\title{
A Systematic Review and Meta-analysis of the Impact of Mindfulness-Based Interventions on the Well-Being of Healthcare Professionals
}

\author{
Tim Lomas $^{1} \cdot$ Joan Carles Medina ${ }^{2} \cdot$ Itai Ivtzan $^{1} \cdot$ Silke Rupprecht $^{3} \cdot$ Francisco José Eiroa-Orosa $^{2}$
}

Published online: 29 November 2018

(C) Springer Science+Business Media, LLC, part of Springer Nature 2018

\begin{abstract}
Efforts to improve the well-being of healthcare professionals include mindfulness-based interventions (MBIs). To understand the value of such initiatives, we conducted a systematic review and meta-analysis of empirical studies pertaining to the use of MBIs with healthcare professionals. Databases were reviewed from the start of records to January 2016 (PROSPERO registration number: CRD42016032899). Eligibility criteria included empirical analyses of well-being outcomes acquired in relation to MBIs. Forty-one papers met the eligibility criteria, consisting of a total of 2101 participants. Studies were examined for two broad classes of well-being outcomes: (a) "negative" mental health measures such as anxiety, depression, and stress; (b) "positive" indices of well-being, such as life satisfaction, together with outcomes associated with well-being, such as emotional intelligence. MBIs were generally associated with positive outcomes in relation to most measures (albeit with moderate effect sizes), and mindfulness does appear to improve the well-being of healthcare professionals. However, the quality of the studies was inconsistent, so further research is needed, particularly high-quality randomised control trials.
\end{abstract}

Keywords Mindfulness $\cdot$ Meditation $\cdot$ Healthcare professionals $\cdot$ Meta-analysis

A wealth of research has accumulated indicating that healthcare professionals (HCPs) are liable to a range of mental health issues, including anxiety (Gao et al. 2012) and depression (Givens and Tjia 2002). These problems may be particularly acute among HCPs relative to other professions: a recent survey of over 3700 public sector workers in the UK found that staff working for the National Health Service were the most stressed, with $61 \%$ reporting feeling stressed all or most of the time and 59\% stating

Electronic supplementary material The online version of this article (https://doi.org/10.1007/s12671-018-1062-5) contains supplementary material, which is available to authorized users.

Francisco José Eiroa-Orosa

fjeiroa@gmail.com; feiroa@ub.edu

1 School of Psychology, University of East London, Arthur Edwards Building, Water Lane, London E15 4LZ, UK

2 Section of Personality, Evaluation and Psychological Treatment, Department of Clinical Psychology and Psychobiology, School of Psychology, Institute of Neurosciences, Universitat de Barcelona, Passeig de la Vall d'Hebron, 171, 08035 Barcelona, Spain

3 Leuphana University, Scharnhorststraße 1, 21335 Lüneburg, Germany that their stress is worse this year than last year (Dudman et al. 2015). These issues represent a significant problem, obviously for the well-being of the HCPs themselves, but also for patients (e.g. the ability of HCPs to treat them skilfully) and for the healthcare system (e.g. the economic cost of staff burnout) (Toppinen-Tanner et al. 2005). As such, efforts are underway to protect against or ameliorate work-related mental health issues in HCPs. Prominent among such initiatives are programmes based around mindfulness meditation - mindfulness-based interventions (MBIs) - which is the focus of this review.

Originating in the context of Buddhism around the 5th century B.C.E. (Lomas 2017), mindfulness came to prominence in the West through Kabat-Zinn's (1982) mindfulnessbased stress reduction (MBSR) programme for chronic pain. "Mindfulness" is frequently used to refer to both (1) a state/ quality of mind and (2) a form of meditation that enables one to cultivate this particular state/quality. (Meditation is a broad label for mental activities which share a common focus on training the self-regulation of attention and awareness, with the goal of enhancing voluntary control of mental processes, thereby increasing well-being (Walsh and Shapiro 2006).) The most prominent operationalisation of mindfulness as a state/ quality of mind is Kabat-Zinn's (2003) definition, which 
constructs it as "the awareness that arises through paying attention on purpose, in the present moment, and nonjudgmentally to the unfolding of experience moment by moment" ( $p$. 145) The term mindfulness is then also deployed for meditation practices which can facilitate this mindful state/quality of mind.

In theoretical terms, the main significance of mindfulness is that it is thought to facilitate a meta-cognitive mechanism known as "decentring"-or alternatively "reperceiving" (Shapiro et al. 2006) - defined as "the ability to observe one's thoughts and feelings as temporary, objective events in the mind, as opposed to reflections of the self that are necessarily true" (Fresco et al. 2007, p. 234). For example, in mindfulness-based cognitive therapy (MBCT) - designed to prevent depressive relapse (Segal et al. 2002) - participants are taught to decentre from their cognitions, thus helping prevent a "downward spiral" of negative thoughts and worsening negative affect which could otherwise precipitate relapse. Thus, MBCT, and MBIs generally, involve "retraining awareness" so that people have greater choice in how they relate and respond to their subjective experience, rather than habitually responding in maladaptive ways (Chambers et al. 2009 , p. 659). The value of this extends across diverse mental health issues. For instance, the development of decentring capabilities can help people tolerate otherwise distressing qualia, which is important given that inability to tolerate such qualia is regarded as a transdiagnostic factor underlying diverse psychopathologies (Aldao et al. 2010).

MBIs were generally limited to clinical populations initially. However, there has been increasing use of mindfulness in occupational contexts, not only for staff who may be suffering with mental health issues, but for workers "in general" (e.g. as a prophylactic against future issues). This emergent literature has been summarised in a raft of recent reviews. These include systematic reviews focusing on specific occupations, including educators (e.g. Emerson et al. 2017; Hwang et al. 2017; Lomas et al. 2017a), social workers (Trowbridge and Mische Lawson 2016), and athletes (Bühlmayer et al. 2017; Noetel et al. 2017), as well as more all-encompassing reviews, such as Lomas, Medina, Ivtzan, Rupprecht, Hart et al. (2017), which included 153 papers across all occupational spheres. These have been augmented by several meta-analyses of non-clinical populations of working adults, such as Virgili (2015) and Khoury et al. (2015). Amidst this general interest in the impact of mindfulness in occupational settings, there is a burgeoning literature focusing on HCPs specifically. This literature has already been summarised in a number of systematic reviews. These include reviews focused on specific sectors and professions, including nurses (Guillaumie et al. 2017), occupational therapists (Luken and Sammons 2016), mental health professionals (Rudaz et al. 2017), "hospital providers" (Luken and Sammons 2016), medical students (Daya and Hearn 2017), and healthcare profession students
(McConville et al. 2017), or on specific outcomes, such as empathy and emotional competency (Lamothe et al. 2016). There have also been more general reviews, such as Lomas et al. (2018), who located 81 studies across all HCP sectors and professions, as well as Eby et al. (2017), who provided a qualitative review of 67 studies. Such reviews have already offered a good indication of the value of mindfulness to HCPs, generally showing a beneficial impact with respect to wellbeing outcomes. However, these reviews have perhaps not revealed the full potential of mindfulness with regard to HCPs, nor have they necessarily provided a robust analysis of its utility or of its limits.

With regard to its potential, many studies have limited their focus to mental health, with a particular focus on specific common disorders such as anxiety and depression (e.g. Guillaumie et al. 2017), stress and distress (Daya and Hearn 2017), as well as employment-related conditions like burnout (Luken and Sammons 2016). However, while such outcomes are of course important, they do not give the full picture of well-being. As a construct, well-being is increasingly favoured in academia as a broad, overarching, and multidimensional term, incorporating all the ways in which a person might hope to do or be well (de Chavez et al. 2005; Lomas et al. 2015b). This not only includes mental health (as per the outcomes alluded to above) but also physical health (Larson 1999), social relationships (Bourdieu 1986), and cognitive performance (Tang et al. 2007). For instance, Pollard and Davidson (2001, p. 10) define well-being as "a state of successful performance across the life course integrating physical, cognitive and social-emotional function." Furthermore, well-being can be appraised in either deficit-based "negative" terms or asset-based "positive" terms. With the former, wellbeing consists in the relative absence of some undesirable phenomenon, such as mental health outcomes like anxiety or depression. However, fields like positive psychology have shown that well-being does not only mean the absence of outcomes like anxiety but also the presence of desirable outcomes (Diener 2000), such as "flourishing" (Keyes 2002) or "satisfaction with life" (Diener et al. 1985). The reviews of the HCP literature cited above generally restrict themselves to deficit-based mental health outcomes, as alluded to above, as indeed do many of the individual studies included within these reviews. There are some exceptions; for instance, both McConville et al. (2017) and Lamothe et al. (2016) included a focus on empathy within their systematic reviews. On the whole though, apart from Lomas et al. (2018), the reviews have not included an expansive look at all facets of well-being, which is something the current paper aims to redress.

The second limitation with the HCP reviews above is that they have not necessarily provided a robust analysis of the utility of mindfulness with respect to this population, nor of its limits. This comment is not a criticism of the reviews per se, but rather a reflection of the inherent analytical limits of reviews, even 
systematic ones. Even though reviews such as Lomas et al. (2018) have sought to calculate and report on effect sizes with respect to the studies reviewed, it is still hard to gain an overall impression of the impact of mindfulness on a particular outcome (other than, for instance, simply reporting on the number of studies that have found a small, medium, or large effect size, or alternatively no effect). For that kind of comparative statistical assessment, metaanalyses are required. Unfortunately, though, to date there have been few meta-analyses focusing on HCPs, and these have been relatively limited in scope. We were only able to locate one that focused on HCPs specifically, an analysis by Burton et al. (2017) which looked just at stress, and featured only seven studies. To this end, the present paper sought to provide a more inclusive meta-analysis of mindfulness in a HCP context, one not limited to particular mental health outcomes such as stress (as per Burton et al. 2017), but rather that takes an inclusive look at the panoply of outcomes pertaining to well-being. The paper is a follow-up to the general systematic review of HCPs provided by Lomas et al. (2018), who located 81 studies across all HCP sectors; of these 81 studies, 37 were selected as being amenable to meta-analysis, as outlined below.

\section{Method}

\section{Eligibility Criteria}

Our analysis considered any study examining the pre-post or controlled effects of MBIs in HCP populations, for a wide range of well-being outcomes, including (a) "negative" mental health measures such as anxiety and depression and (b) "positive" indices of well-being, such as life satisfaction, including outcomes associated with well-being, such as emotional intelligence. The literature search was conducted using the MEDLINE and Scopus electronic databases; terms included in the review were mindfulness AND work OR occupation OR profession OR staff (in all fields in MEDLINE and limited to article title, abstract, and keywords in Scopus).

\section{Search Strategies}

The search was conducted as part of a broader ongoing systematic review on mindfulness in all occupations (please see Lomas, Medina, Ivtzan, Rupprecht, Hart, and Eiroa-Orosa, 2017). The dates selected were from the start of the database records to 10th January 2016. We also looked through the reference lists of studies selected for inclusion in the review for other articles that may be relevant (but which did not appear in our database search). For the current review of HCPs specifically, in terms of PICOS (participants, interventions, comparisons, outcomes, and study design), the key inclusion criteria were participants - currently employed in a healthcare context; outcomes - any pertaining to well-being (using this term in the broad, inclusive way outlined above); and study design - any empirical study examining the quantitative prepost or controlled effects of MBIs in HCP populations.

\section{Inclusion and Exclusion Criteria}

Exclusion criteria were theoretical articles, commentaries without statistical analyses, and studies that did not feature pre-post quantitative testing of an MBI. Studies were required to be published (or in press) in English in a peer-reviewed academic journal. The review was conducted according to the Preferred Reporting Items for Systematic Reviews and Meta-Analyses (PRISMA) guidelines (Moher et al. 2009). The review protocol was registered with the International Prospective Register of Systematic Reviews (PROSPERO) database on 5th January 2016 (registration number: CRD42016032899).

\section{Data Extraction}

The following variables were extracted from each paper: type of design (i.e. randomised controlled trial [RCT] versus prepost and non-randomised intervention studies); occupation of participants; number of experimental participants; number of control participants and nature of the control condition (if applicable); type of MBI; length of MBI; well-being outcomes; and the mean and standard deviations of principle outcomes.

As discussed above, well-being serves as an allencompassing, multidimensional construct that includes all the ways a person might hope to do or be well (de Chavez et al. 2005). In this review, two "classes" of well-being measures were extracted. First, the main measures were psychometric scales pertaining to "deficit-based" mental health outcomes - i.e. whose relative absence is regarded as indicative of well-being, as elucidated above - such as anxiety and depression. Second, there were various positive "asset-based" psychological outcomes-i.e. whose relative presence is regarded as indicative of well-being - such as satisfaction with life. This second class included outcomes that, although not regarded as indices of well-being per se, are closely associated with it, such as emotional intelligence (Salovey and Mayer 1990). Whenever a study met the inclusion criteria to be part of the meta-analysis but did not report all the data needed to compute weighted parameters, trial authors were contacted to request all the missing information.

\section{Quality Assessment}

The Quality Assessment Tool for Quantitative Studies (QATQS; National Collaborating Centre for Methods and Tools 2008) was used to assess the quality of the studies. QATQS assesses methodological rigour in six areas: (a) selection bias; (b) design; (c) confounders; (d) blinding; (e) data 
collection method; and (f) withdrawals and drop-outs. Each area is assessed on a quality score of 1 to 3 ( $1=$ strong; $2=$ moderate; 3 = weak). Scores for each area were collated and a global score assigned to each study. If there are no weak ratings, the study is scored 1 (strong); one weak rating leads to a 2 (moderate); and two or more weak ratings generates a 3 (weak). QATQS scoring was conducted primarily by the third author, following the guidelines outlined in the QATQS protocol. While not specifically in receipt of QATQS training, the author is a senior lecturer in psychology with over 15 years of active research experience, including with respect to conducting systematic reviews, and with respect to mindfulness specificallysee Lomas et al. (2015c) for an example of previous work in this regard) - of which he is also an experienced teacher and teacher trainer. A sample of 15 papers was independently coded by the first author; while also not specifically trained in QATQS coding, he is a senior lecturer in psychology with over 8 years of active research experience, including with respect to conducting systematic reviews of mindfulness specifically (as per Lomas et al. 2015c). There was a disagreement only with respect to one paper, where the first author disagreed with the scores for three of the QATQS criteria assigned by the third author. These discrepancies were resolved by discussion (with an amended score accepted on one of the criteria). In light of that discussion, the third author re-checked the rest of the papers, but this did not lead to any further revisions in coding.

\section{Statistical Analyses}

The meta package (Schwarzer 2007) for the R software ( $R$ Core Team 2017) was used to compute the statistical analyses and create funnel and forest plots. As we were assessing studies carried with different formats in different contexts, we chose random effects models as we assumed that the estimates of treatment effect could vary across studies because of real differences in the intervention effect (Riley et al. 2011). Only outcomes represented in three or more studies are included in the models and, therefore, forest plots, although all outcomes for all studies were included in the analyses for publication bias. We assessed publication bias using contour-enhanced funnel plots and Begg and Mazumdar's (1994) tests by outcome valence. In cases where a study reported a trial with two intervention groups and at least one control group, separate analyses were conducted for each inter-group comparison.

As most studies reported means and standard deviations, according to the aforementioned variable grouping strategy, different scales were grouped under a common outcome type. We calculated Hedges' $g$ standardised mean differences with $95 \%$ confidence intervals (Sedgwick and Marston 2013) for each outcome within each study design. When adding a negative valence scale to an asset-based outcome, means were recoded (multiplied by minus one) so that the valences coincided. For studies with more than one scale in the same outcome group, mean values for each of these metrics were converted to a single mean value for the intervention and control groups respectively. The variance of the mean among scales included within the same outcome grouping was calculated using Borenstein, Hedges, Higgins, and Rothstein's method (Borenstein et al. 2009):

$\operatorname{var}\left(\frac{1}{m} \sum_{i=1}^{m} Y_{i}\right)=\left(\frac{1}{m}\right)^{2}\left(\sum_{i=1}^{m} V_{i}+\sum_{i \neq j}\left(r_{i j} \sqrt{V_{i}} \sqrt{V_{j}}\right)\right)$

When the correlation between scales was unknown, $r=.5$ was assumed as a midpoint between total independence and total dependence. This procedure was implemented to estimate all outcomes' overall effect size, confidence intervals, sample size, and heterogeneity and was needed to preserve the statistical independence of assumptions, controlling for the risk of bias due to the inflation of the main effect size's variance.

Heterogeneity was systematically assessed among the studies using the Cochran's $Q, I^{2}$, and the $\tau^{2}$ statistics. While Cochran's $Q$ (a chi-squared distributed measure of weighted squared deviations that can be converted into a $p$ value) is the usual test statistic, the principal advantage of $I^{2}$ (the ratio of true heterogeneity to total observed variation, i.e. the proportion of the observed variance reflecting real differences in effect size) is that it can be calculated and compared across metaanalyses of different sizes, of different types of study, and using different types of outcome data (Higgins et al. 2003). $\tau^{2}$ is the variance of the true effect sizes (i.e. the actual standard deviation), calculated as part of random effects meta-analyses.

Finally, to account for possible moderators, all covariates that can usually be found in similar meta-analyses (Khoury et al. 2013; Spielmans \& Flückiger 2018) and were possible to gather within the studies analysed were taken into account: study design type (non-randomised trials/quasi-experimental designs, pre-post studies, RCTs); publication year of the study; gender; age; profession (students vs. professionals); type of intervention (MBSR vs. others); treatment intensity (including a compound outcome made of treatment duration, session length, homework, retreatments, and frequency); professional activity; and studies' QATQS scores. These factors were all correlated with metaanalytic models using tests for subgroup differences and metaregressions. These analyses were performed taking each outcome as a unit, as doing it within each study design would mean lacking an adequate sample for practically all calculations.

\section{Results}

\section{Literature Search Results}

For the broader systematic review-i.e. mindfulness across all occupations (Lomas, Medina, Ivtzan, Rupprecht, Hart, and Eiroa-Orosa, 2017) - following the removal of duplicate citations, 721 potentially relevant papers were identified. In the current 
systematic review, focusing specifically on $\mathrm{HCPs}$, from reviewing the abstract, 543 papers were excluded, while from the full text reviews of 178 papers, 124 further papers were also excluded. From the 54 articles within the scope of this review, 13 were not included in the analysis since they were qualitative studies, therefore leaving 41 articles. However, since inclusion in the analyses required that study designs with a specific outcome had to have been assessed by at least three different studies (Higgins and Green 2011), four studies (Gockel et al. 2013; Grepmair et al. 2007; Poulin et al. 2008 and Singh et al. 2015) were only included in publication bias analyses. This process of winnowing is shown below as a PRISMA flow diagram (see Fig. 1).

The studies comprised a total of 2101 participants (discounting participants not including in analyses due to attrition), including 1415 undertaking MBIs and 686 separate control participants. The studies $(n=42$, as one study, Goodman and Schorling 2012; had a sample of physicians and another of other providers, considered separately for analyses) covered a range of occupations, including healthcare students $(n=15)$, physicians $(n=5)$, nurses $(n=6)$, therapists, mental health $(n=$ 5 ), and mixed (non-specific) healthcare professionals $(n=11)$. As for study design, 24 were pre-post studies of a single sample, 12 RCTs, and 6 non-randomised studies. Details on effect sizes of the particular studies - which have also been previously described in Lomas, Medina, Ivtzan, Rupprecht, Hart, and EiroaOrosa (2017) - are outlined below in Table 1, and a summary of the overall statistically significance of outcomes change is shown in Table 2. In Table 2, studies have been grouped according to the specific well-being outcomes they explicitly reported on. In most cases, particularly with respect to "deficit-based" outcome measures, studies reported on well-established common constructs (e.g. anxiety, depression, distress, and stress). In some instances, though, outcomes which were less-frequently reported on have been aggregated into larger categories. For instance, a heterogenous range of "positive" measures were reported by a number of studies, such as satisfaction with life and positive affect, and these have been aggregated into a category of "positive well-being." In addition, Table 3 shows the outcomes of the QATQS quality assessment.

\section{Reporting Bias}

We constructed two contour-enhanced funnel plots by grouping positive (e.g. satisfaction) and negative (e.g. distress) outcome measures (see Figs. 2 and 3). Singh et al. (2006) and Singh et al. (2015) were excluded from the funnel plots due to extreme SMD values (28.98 and -3.89 respectively), and Begg and Mazumdar's (1994) tests were calculated both including and excluding them. Both funnel plots showed an apparently symmetric distribution. When testing asymmetry with Begg and Mazumdar's tests, both positive $(z=-0.623, p=.53$; $z=-0.238, p=.81$, including Singh et al. 2006) and negative

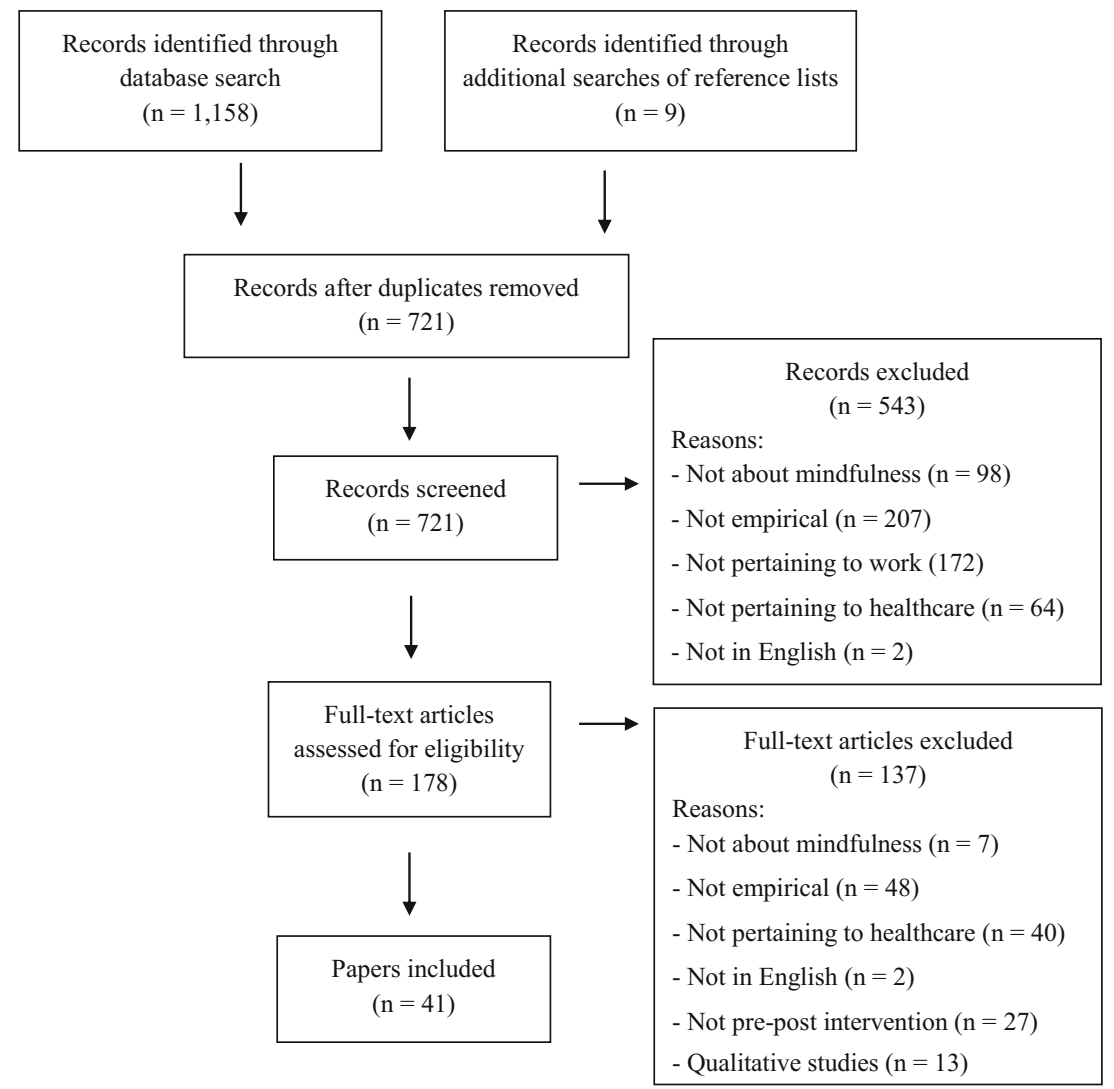

Fig. 1 The Preferred Reporting Items for Systematic Reviews and Meta-Analyses (PRISMA) flow diagram 


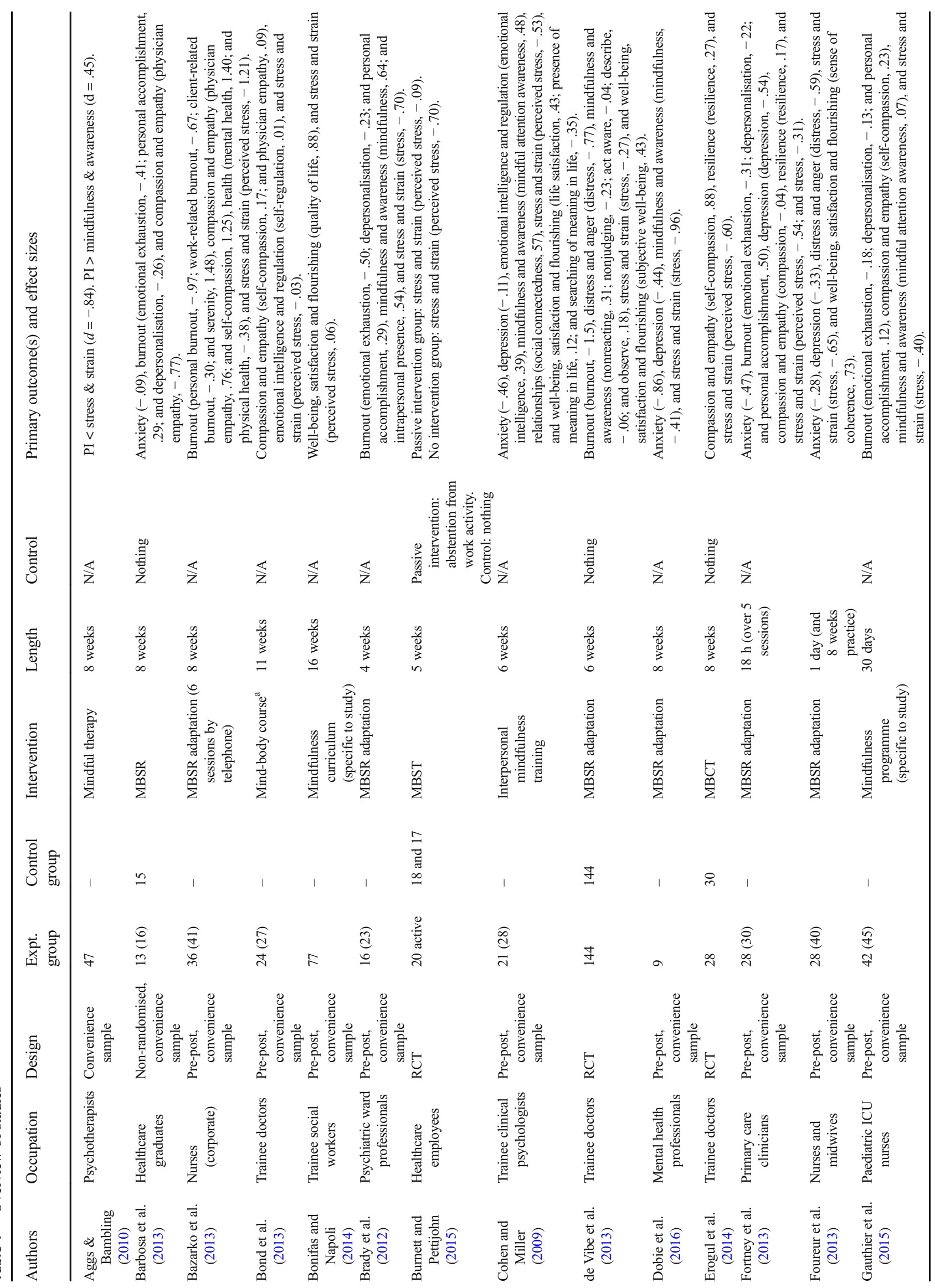




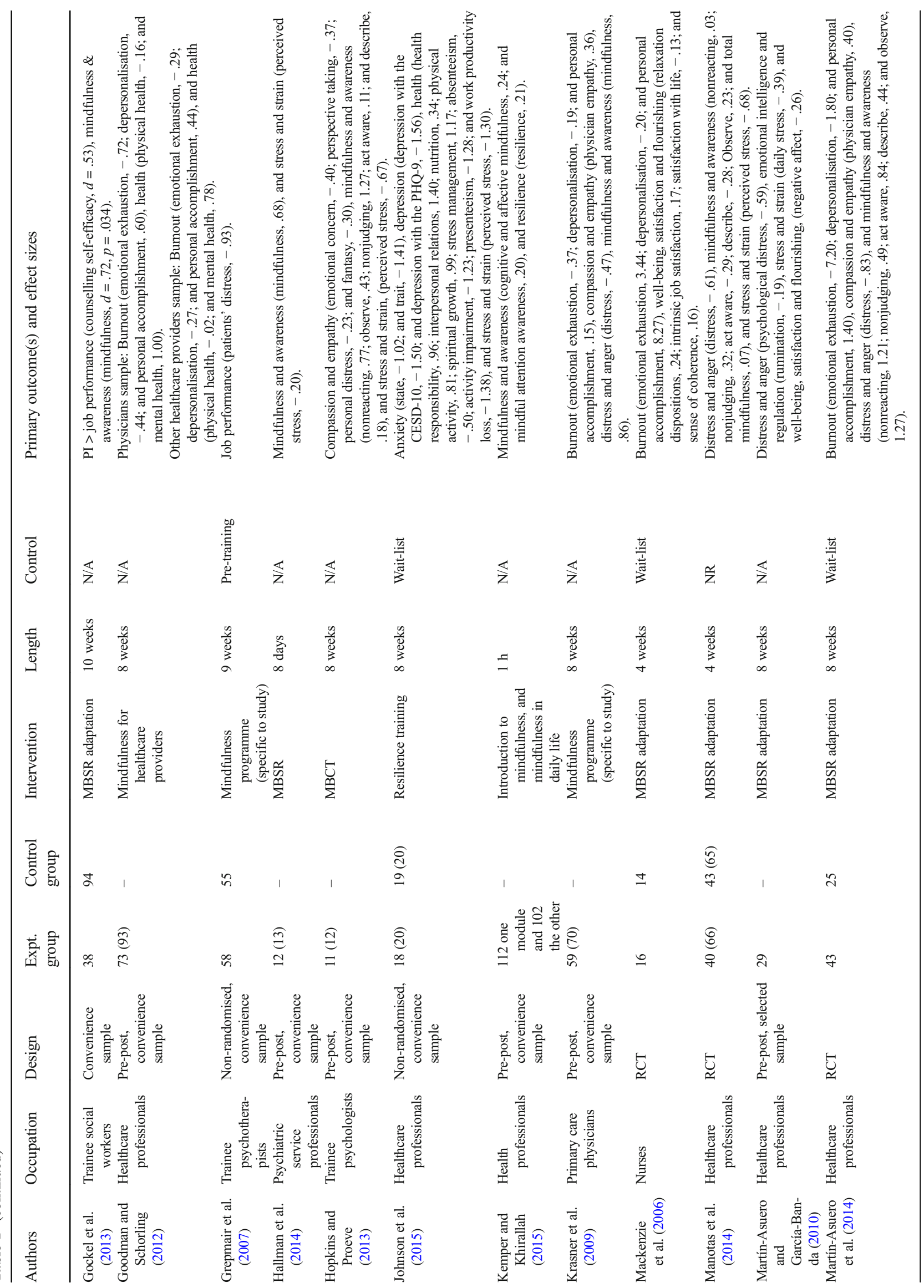




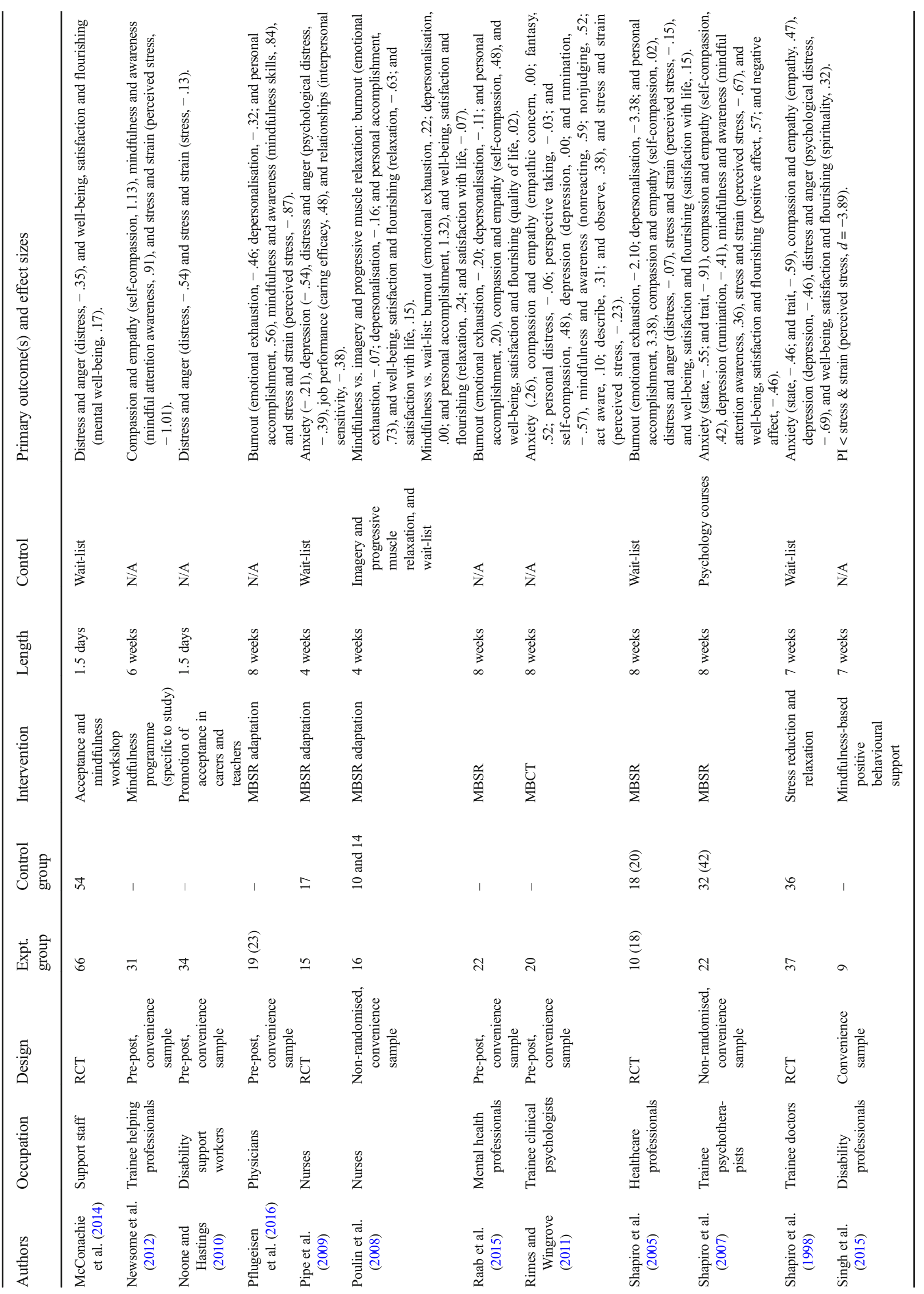


$(z=-0.792, p=0.43 ; z=-1.113, p=.27$, including Singh et al. 2015) outcomes showed no statistically significant asymmetry.

\section{"Negative" Well-Being Outcomes}

Anxiety Mindfulness appears to have a beneficial impact upon anxiety (which was the only dependent variable with enough studies to perform calculations in all three design types), as shown in Fig. 4 below. Effect sizes for non-randomised trials, pre-post studies and RCTs were $-1.01(95 \% \mathrm{CI}=-2.06$, $0.04, p=.059),-0.31(95 \% \mathrm{CI}=-0.62,-0.01, p<0.05)$ and $-0.49(95 \% \mathrm{CI}=-0.81,-0.16, p<0.005)$ respectively, with most studies showing a reduction in anxiety as a result of the intervention. High and statistically significant heterogeneity was found just for non-randomised trials $\left(I^{2}=85 \%\right.$, $\left.\tau^{2}=.724, \chi^{2}=13.19, p<.001\right)$. No statistically significant results were found for any moderator (and calculations could not be carried using MSBR or homework as independent variable, as only one study did not use this intervention model in its implementation, and all studies included take-home activities).

Burnout Mindfulness appears to have a beneficial impact upon burnout, as shown in Fig. 5 below, with effect sizes of $0.51(95 \% \mathrm{CI}=-0.70,-0.32, p<.0001)$ for pre-post studies and $-0.31(95 \% \mathrm{CI}=-0.57,-0.04, p=.024)$ for RCTs. Heterogeneity was not statistically significant in both cases. In addition, one study (Mackenzie et al. 2006) had significant differences between groups at pre-intervention time, which needs to be taken into account when interpreting the results. No statistically significant results were found for any moderator or between study designs.

Depression Mindfulness appears to have a beneficial impact upon depression, as shown in Fig. 6 below, with effect sizes of $-0.29(95 \% \mathrm{CI}=-0.55,-0.03, p<.05)$ for pre-post designs and $-0.55(95 \% \mathrm{CI}=-0.87,-0.22, p=.001)$ for RCTs. In these analyses, neither heterogeneity nor subgroup differences showed statistical significance. No statistically significant results were found for any moderator or between study designs.

Distress Mindfulness appears to have a beneficial impact upon distress and anger, as shown in Fig. 7 below, with effect sizes of $-0.54(95 \% \mathrm{CI}=-0.75,-0.33, p<.0001)$ for pre-post and $-0.61(95 \% \mathrm{CI}=-0.79,-0.44, p<.0001)$ for RCTs. Neither heterogeneity nor the design subgroup differences or any moderator showed statistically significant differences.

Stress Mindfulness appears to have a beneficial impact upon stress, as shown in Fig. 8 below, with effect sizes of -0.58 $(95 \% \mathrm{CI}=-0.81,-0.34, p<.0001)$ for pre-post and -0.42 $(95 \% \mathrm{CI}=-0.67,-0.17, p=.0001)$ for RCTs. High and 
Table 2 Summary of statistically significance of common outcomes across all studies

\begin{tabular}{lrll}
\hline Outcome & $\begin{array}{l}\text { Number of } \\
\text { studies assessing }\end{array}$ & $\begin{array}{l}\text { Statistical improvement } \\
\text { related to mindfulness } \\
\text { intervention }\end{array}$ & $\begin{array}{l}\text { No statistically significant } \\
\text { change in relation to } \\
\text { mindfulness intervention }\end{array}$ \\
\hline Anxiety & 11 & 10 & 1 \\
Burnout & 14 & 7 & 7 \\
Compassion & 12 & 9 & 3 \\
Depression & 9 & 4 & 5 \\
Distress & 13 & 11 & 2 \\
Emotional regulation & 3 & 0 & 3 \\
Empathy & 7 & 6 & 1 \\
Health & 3 & 3 & 0 \\
Job performance & 4 & 3 & 4 \\
Mindfulness & 19 & 15 & 8 \\
Stress & 27 & 19 & 7 \\
Positive well-being & 14 & 7 & 4 \\
\hline
\end{tabular}

${ }^{\text {a }}$ The studies included in this category showed no statistically significant improvements in the given outcome. None of the studies showed statistically significant deteriorations statistically significant heterogeneity was found just for prepost designs $\left(I^{2}=66 \%, \tau^{2}=.154, \chi^{2}=50.5, p<.0001\right)$, but the subgroup differences were not. Additionally, one study included here (Burnett and Pettijohn 2015) observed significant pre-intervention differences between the two groups; hence, its results must be interpreted with caution. No statistically significant results were found for any moderator.

\section{"Positive" Well-Being Outcomes}

Compassion Mindfulness appears to have a beneficial impact upon compassion, as shown in Fig. 9 below, with effect sizes of $0.52(95 \% \mathrm{CI}=0.15,0.90, p=.006)$ for pre-post and $0.35(95 \% \mathrm{CI}=-0.08,0.78, p=.109)$ for RCTs (although the latter was not statistically significant). Both had high heterogeneity levels, but statistical significance was only reached with pre-post designs $\left(I^{2}=71 \%\right.$, $\tau^{2}=.181, \chi^{2}=20.93, p=.002$ ). Hence, again, results should be interpreted with caution. Statistically significant higher effect sizes were found in studies carried using the original MSBR $(Q=4.53, p<.05)$ and including retreatments $(Q=$ $5.22, p<.05)$. Calculations could not be carried using homework as independent variable as all studies included take-home activities.

Emotional Intelligence and Regulation There was only enough information to perform meta-analytic calculations for pre-post designs with this variable. In contrast to other outcomes, the results showed no significant differences in emotional intelligence and regulation after mindfulness practice. As Fig. 10 displays, although there was a mild improvement, it did not reach statistical significance, with an overall effect size of $0.18(95 \% \mathrm{CI}=-0.14,0.51, p=$
0.26). The level of heterogeneity was non-significant. No statistically significant results were found for any moderator.

Empathy As in the case of emotional intelligence, only prepost designs were numerous enough to perform calculations. Mindfulness appears to have a beneficial impact upon empathy, as shown in Fig. 11 below, with an effect size of 0.31 (95\% CI $=0.02,0.60, p<.05)$. Heterogeneity and subgroup differences were non-significant, and no statistically significant correlations were found with any moderator.

Positive well-being Mindfulness appears to have a beneficial impact upon "positive well-being" (e.g. life satisfaction), as shown in Fig. 12 below, with effect sizes of 0.49 (95\% CI = $0.14,0.83, p=.005)$ for pre-post and $0.27(95 \% \mathrm{CI}=0.12$, $0.43, p<.001)$ for RCTs. With pre-post designs, the heterogeneity was statistically significant $\left(I^{2}=58 \%, \tau^{2}=.088, \chi^{2}=\right.$ $9.59, p=.05)$. Subgroup differences were non-significant. Statistically significant correlations were found for intervention intensity $(\mathrm{QM}=4.718, p<.05)$ with higher gains for more intense interventions and for profession $(Q=4.18$, $p<.05)$ with higher gains for students.

Mindfulness Mindfulness practice appears to have a beneficial impact upon mindfulness, as shown in Fig. 13 below, with effect sizes of $0.52(95 \% \mathrm{CI}=0.31,0.73, p<.0001)$ for pre-post and $0.34(95 \% \mathrm{CI}=-0.06,0.73, p=.09)$ for RCTs (although the latter was not statistically significant). Heterogeneity was relatively high and statistically significant in both cases (pre-post: $I^{2}=46 \%, \tau^{2}=.056, \chi^{2}=20.29$, $p=.04$, RCTs: $I^{2}=72 \%, \tau^{2}=.136, \chi^{2}=14.3, p<.01$ ), but subgroup differences were not. Statistically significant 
Table 3 QATQS scoring assessment of studies

\begin{tabular}{|c|c|c|c|c|c|c|c|}
\hline Authors & Selection bias & Design & Cofounders & blinding & Data collection & Attrition & Global \\
\hline Aggs \& Bambling (2010) & 3 & 3 & 3 & 3 & 3 & 2 & 3 \\
\hline Barbosa et al. (2013) & 3 & 3 & 2 & 3 & 2 & 2 & 3 \\
\hline Bazarko et al. (2013) & 2 & 3 & 3 & 3 & 2 & 1 & 3 \\
\hline Bond et al. (2013) & 3 & 3 & 3 & 3 & 2 & 2 & 3 \\
\hline Bonifas and Napoli (2014) & 3 & 3 & 3 & 3 & 1 & 1 & 3 \\
\hline Brady et al. (2012) & 3 & 3 & 3 & 3 & 2 & 2 & 3 \\
\hline Burnett and Pettijohn (2015) & 3 & 2 & 2 & 3 & 1 & 2 & 3 \\
\hline Cohen and Miller (2009) & 3 & 3 & 3 & 3 & 1 & 2 & 3 \\
\hline de Vibe et al. (2013) & 2 & 1 & 1 & 1 & 1 & 1 & 1 \\
\hline Dobie et al. (2016) & 3 & 3 & 3 & 3 & 1 & 1 & 3 \\
\hline Erogul et al. (2014) & 1 & 1 & 1 & 1 & 1 & 2 & 1 \\
\hline Fortney et al. (2013) & 3 & 3 & 3 & 3 & 1 & 1 & 3 \\
\hline Foureur et al. (2013) & 3 & 3 & 3 & 3 & 1 & 2 & 3 \\
\hline Gauthier et al. (2015) & 3 & 3 & 3 & 3 & 1 & 2 & 3 \\
\hline Gockel et al. (2013) & 3 & 3 & 3 & 3 & 1 & 2 & 3 \\
\hline Goodman and Schorling (2012) & 2 & 3 & 3 & 3 & 1 & 3 & 3 \\
\hline Grepmair et al. (2007) & 2 & 2 & 1 & 2 & 1 & 1 & 2 \\
\hline Hallman et al. (2014) & 2 & 3 & 3 & 2 & 1 & 1 & 3 \\
\hline Hopkins and Proeve (2013) & 3 & 3 & 3 & 3 & 1 & 2 & 3 \\
\hline Johnson et al. (2015) & 3 & 3 & 1 & 3 & 1 & 2 & 3 \\
\hline Kemper and Khirallah (2015) & 3 & 3 & 3 & 3 & 1 & 2 & 3 \\
\hline Krasner et al. (2009) & 2 & 3 & 3 & 3 & 1 & 2 & 3 \\
\hline Mackenzie et al. (2006) & 3 & 2 & 2 & 3 & 1 & 2 & 3 \\
\hline Manotas et al. (2014) & 2 & 2 & 1 & 3 & 1 & 2 & 2 \\
\hline Martín-Asuero and García-Banda (2010) & 2 & 3 & 3 & 3 & 1 & 1 & 3 \\
\hline Martín-Asuero et al. (2014) & 2 & 2 & 2 & 3 & 1 & 1 & 2 \\
\hline McConachie et al. (2014) & 2 & 1 & 1 & 3 & 1 & 2 & 2 \\
\hline Newsome et al. (2012) & 1 & 3 & 3 & 2 & 1 & 2 & 3 \\
\hline Noone and Hastings (2010) & 1 & 3 & 3 & 1 & 1 & 3 & 3 \\
\hline Pflugeisen et al. (2016) & 1 & 3 & 3 & 2 & 2 & 1 & 3 \\
\hline Pipe et al. (2009) & 2 & 1 & 2 & 1 & 1 & 1 & 1 \\
\hline Poulin et al. (2008) & 1 & 2 & 1 & 1 & 1 & 3 & 2 \\
\hline Raab et al. (2015) & 1 & 3 & 3 & 2 & 1 & 2 & 3 \\
\hline Rimes and Wingrove (2011) & 1 & 3 & 3 & 1 & 1 & 3 & 3 \\
\hline Shapiro et al. (1998) & 1 & 1 & 2 & 1 & 1 & 1 & 1 \\
\hline Shapiro et al. (2005) & 1 & 1 & 2 & 2 & 1 & 3 & 2 \\
\hline Shapiro et al. (2007) & 1 & 2 & 1 & 2 & 1 & 1 & 1 \\
\hline Singh et al. (2015) & 1 & 3 & 3 & 1 & 1 & 3 & 2 \\
\hline Singh et al. (2006) & 1 & 3 & 3 & 1 & 2 & 2 & 3 \\
\hline Song and Lindquist (2015) & 1 & 1 & 2 & 1 & 1 & 1 & 1 \\
\hline West et al. (2014) & 1 & 1 & 1 & 1 & 1 & 2 & 1 \\
\hline
\end{tabular}

correlations were found for intervention intensity $(\mathrm{QM}=$ $4.888, p<.05)$ with higher gains for more intense interventions. Additionally, and contrarily to what we found for compassion, higher effect sizes were found in studies not using the original MSBR $(Q=4.53, p<.05)$.

\section{Discussion}

Overall, MBIs appeared to have a positive impact upon most outcome measures (albeit with moderate effect sizes), of which there was a great range. As discussed above, one of the prerogatives of the current review was to take an inclusive 


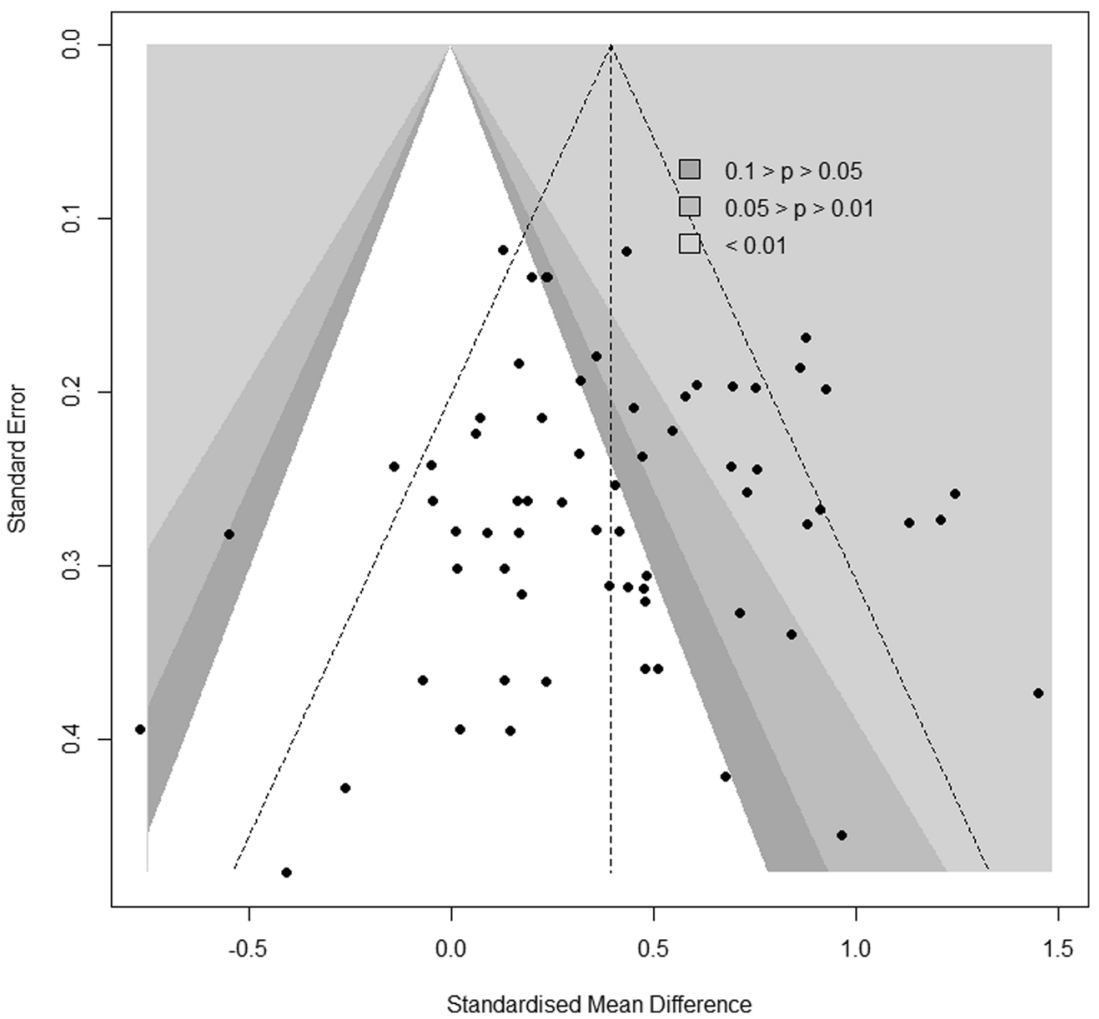

Fig. 2 Funnel plot of positive outcomes. Footnote: funnel plot including the 37 articles fulfilling all inclusion and exclusion criteria

approach to well-being, viewing this as a multidimensional construct encompassing the myriad ways a person might hope to do or be well (de Chavez et al. 2005). Such an approach differentiates the current paper from previous analyses on the

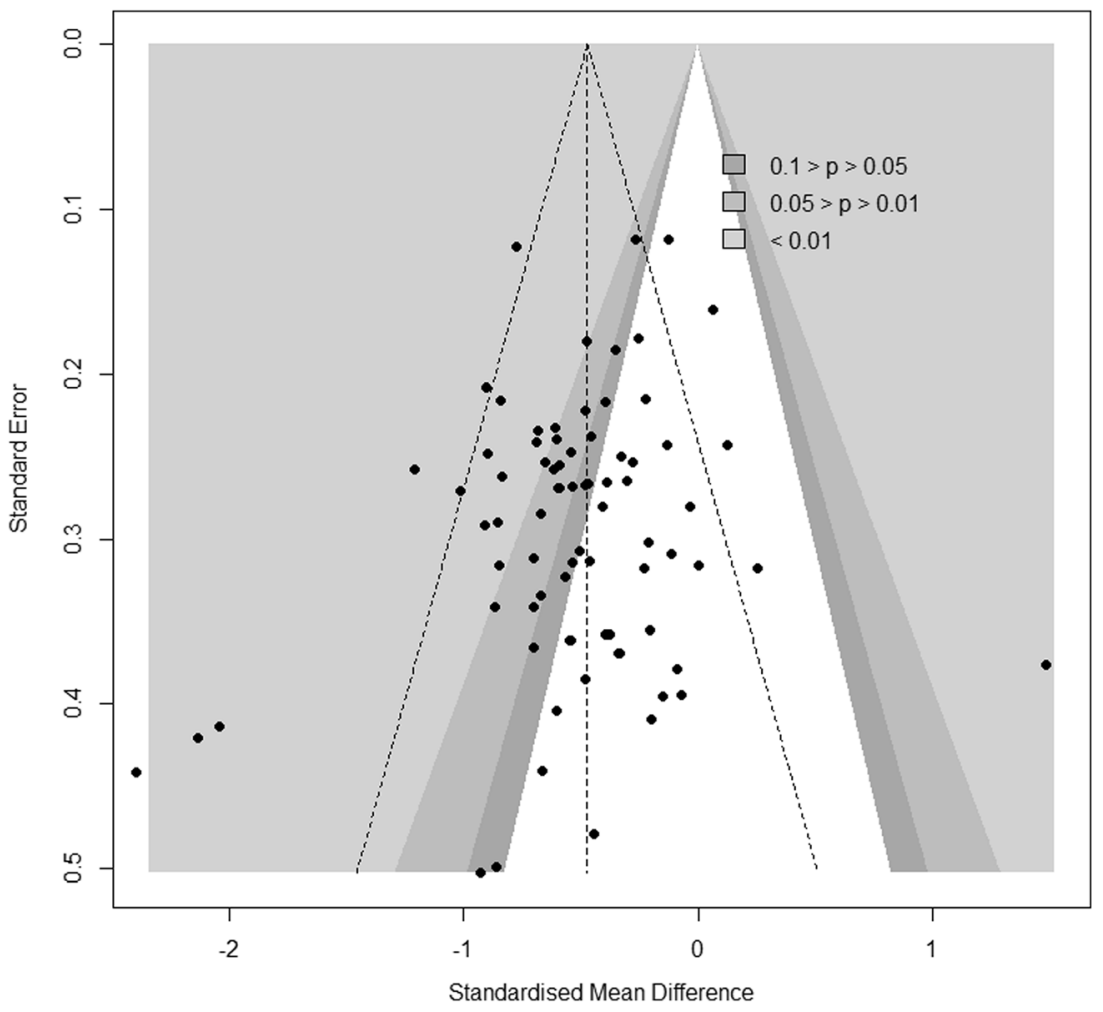

Fig. 3 Funnel plot of negative outcomes. Footnote: funnel plot including the 37 articles fulfilling all inclusion and exclusion criteria 
Study

Experimental / Post Control/Pre Total Mean SD Total Mean SD

\section{Standardised Mean \\ Difference}

SMD

$95 \%-\mathrm{Cl}$
Nonrandomised Trials

Shapiro, Brown, \& Biegel, 2007

Johnson, Emmons, Rivard, Griffin, \& Dusek, 2015

Random effects model

Heterogeneity: $I^{2}=85 \%, \tau^{2}=0.7243, \chi_{2}^{2}=13.19(p<0.01)$

Pre-Post

Cohen \& Miller, 2009

Rimes \& Wingrove, 2011

Fortney, Luchterhand, Zakletskaia, et al., 2013

Foureur, Besley, Burton, Yu, \& Crisp, 2013

Dobie, Tucker, Ferrari, \& Rogers, 2015
Barbosa et al., 2013

Random effects model

Heterogeneity: $I^{2}=20 \%, \tau^{2}=0.0246, \chi_{4}^{2}=5.02(p=0.29)$

RCT

Shapiro, Schwartz, \& Bonner, 1998

Pipe et al., 2009

Song \& Lindquist, 2015

Random effects model

Heterogeneity: $I^{2}=0 \%, \tau^{2}=0, x_{2}^{2}=0.85(p=0.65)$

Fig. 4 Forest plot of anxiety outcomes. Footnote: scale by article in alphabetical order. Barbosa et al. 2013: Burns Anxiety Inventory; Cohen and Miller 2009: Beck Anxiety Inventory; Dobie et al. 2016: Depression, Anxiety and Stress Scales (DASS)-Anxiety; Fortney et al. 2013: DASS-Anxiety; Foureur et al. 2013: DASS-Anxiety; Johnson

impact of mindfulness in HCPs, which have tended to just focus on "deficit-based" mental health outcomes such as anxiety and depression. For instance, the only meta-analysis we located concentrating on HCPs specifically was just Anxiety

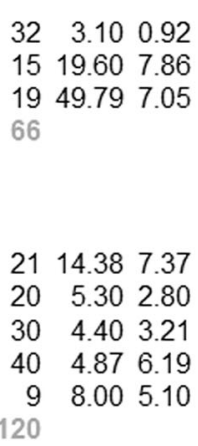

$\begin{array}{rrr}36 & 41.80 & 9.28 \\ 17 & 0.28 & 0.26 \\ 23 & 5.90 & 7.40 \\ 76 & & \end{array}$

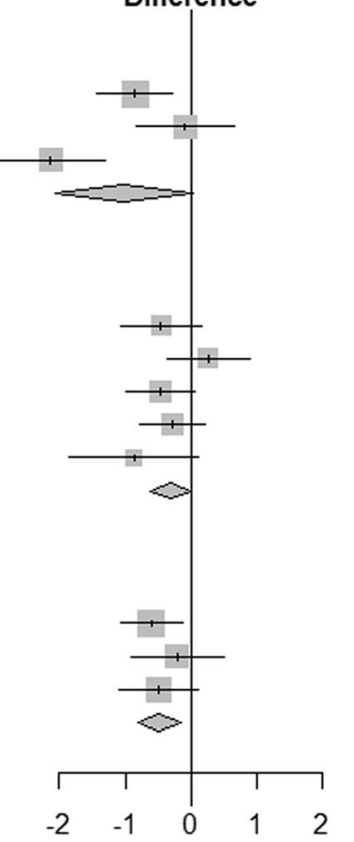

$-0.85[-1.42 ;-0.28]$

$-0.09[-0.83 ; 0.65]$

$-2.14[-2.96 ;-1.31]$

$-1.01[-2.06 ; 0.04]$

$-0.46[-1.08 ; 0.15]$

$0.26[-0.37 ; 0.88]$

$-0.47 \quad[-0.99 ; 0.05]$

$-0.28[-0.78 ; 0.22]$

$-0.86[-1.84 ; 0.11]$

$-0.31[-0.62 ;-0.01]$

$-0.60[-1.07 ;-0.13]$

$-0.21 \quad[-0.90 ; 0.49]$

$-0.50[-1.10 ; 0.10]$

$-0.49[-0.81 ;-0.16]$

et al. 2015: State-Trait Anxiety Inventory (STAI); Pipe et al. 2009: Symptom Checklis t-90-R-Anxiety; Rimes and Wingrove 2011: Hospital Anxiety and Depression Scales-Anxiety; Shapiro et al. 2007: STAI; Shapiro et al. 1998: STAI; Song and Lindquist 2015: DASS-

concerned with stress, featuring only seven studies (Burton et al. 2017). By contrast, the current review looked at two broad classes of well-being outcomes: (a) negative "deficitbased" mental health outcomes (e.g. depression), (b) positive

\section{Control/Pre Standardised Mean}

Experimental / Post

Control/Pre

Study Total Mean SD Total Mean SD

Pre-Post

Krasner et al., 2009

Brady, OConnor, Burgermeister, \& Hanson, 2009

Goodman \& Schorling, 2012 Physicians

Goodman \& Schorling, 2012 Other providers

Bazarko, Cate, Azocar, \& Kreitzer, 2013

Fortney, Luchterhand, Zakletskaia, et al., 2013

Gauthier, Meyer, Grefe, \& Gold, 2015

Raab, Sogge, Parker, \& Flament, 2015

Pflugeisen, Drummond, Ebersole, et al., 2016

Random effects model

Heterogeneity: $I^{2}=28 \%, \tau^{2}=0.0230, \chi_{8}^{2}=11.08(p=0.20)$

$\begin{array}{lll}59 & 24.17 & 7.51\end{array}$

$\begin{array}{lll}16 & -7.27 & 5.26\end{array}$

$\begin{array}{lll}51 & -4.33 & 4.35\end{array}$

$\begin{array}{lll}42 & -6.07 & 4.29\end{array}$

$36 \quad 26.0112 .02$

$\begin{array}{lll}28 & -0.30 & 4.54\end{array}$

$\begin{array}{lll}42 & -0.28 & 4.88\end{array}$

$\begin{array}{lll}22 & -0.79 & 0.59\end{array}$

$\begin{array}{lll}19 & -4.50 & 5.20\end{array}$

315

RCT

Shapiro, Astin, Bishop, \& Cordova, 2005

Mackenzie, Poulin, \& Seidman-Carlson, 2006

de Vibe et al., 2013

Martin-Asuero et al., 2014

Random effects model

Heterogeneity: $I^{2}=22 \%, \tau^{2}=0.0189, \gamma_{3}^{2}=3.87(p=0.28)$

Fig. 5 Forest plot of burnout outcomes. Footnote: scale by article in alphabetical order. Bazarko et al. 2013: Copenhagen Burnout Inventory; Brady et al. 2012: Maslach Burnout Inventory (MBI); De Vibe et al. 2013: MBI-Student version; Fortney et al. 2013: MBI; Gauthier et al.

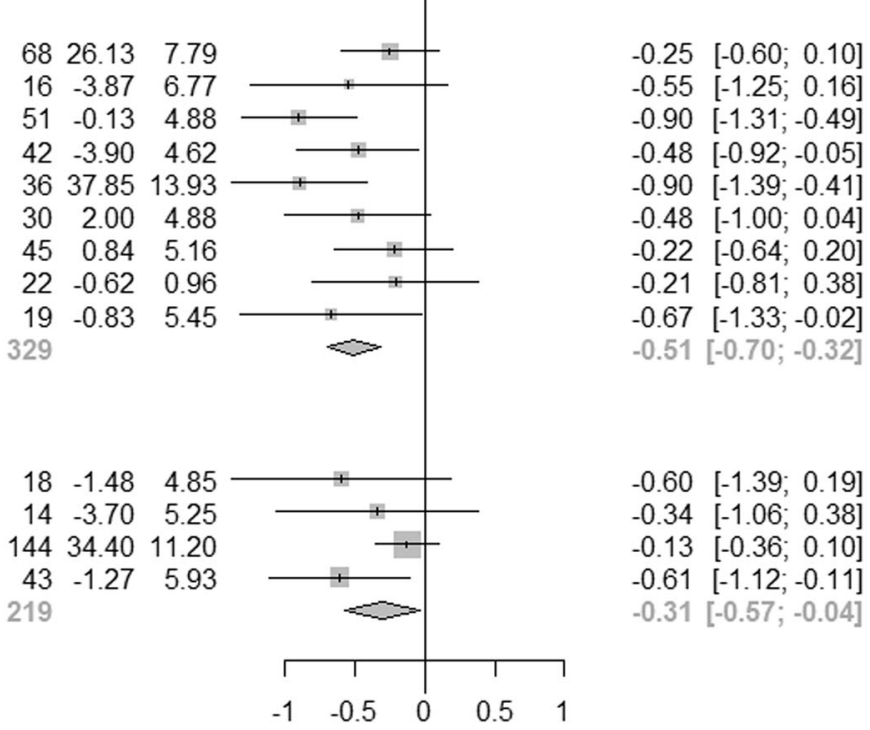

2015: MBI; Goodman and Schorling 2012: MBI; Krasner et al. 2009: MBI; Martín-Asuero et al. 2014: MBI; Mackenzie et al. 2006: MBI; Martín-Asuero et al. 2014: MBI; Pflugeisen et al. 2016: MBI; Raab et al. 2015; MBI; Shapiro et al. 2005: MBI 
Study

Experimental/Post Control/Pre Total Mean SD Total Mean SD
Pre-Post

Cohen \& Miller, 2009

Rimes \& Wingrove, 2011

Fortney, Luchterhand, Zakletskaia, et al., 2013

Foureur, Besley, Burton, Yu, \& Crisp, 2013

Dobie, Tucker, Ferrari, \& Rogers, 2015

Random effects model

Heterogeneity: $I^{2}=0 \%, \tau^{2}=0, \chi_{4}^{2}=2.17(p=0.70)$

RCT

Shapiro, Schwartz, \& Bonner, 1998

Pipe et al., 2009

Song \& Lindquist, 2015

Random effects model

Heterogeneity: $I^{2}=0 \%, \tau^{2}=0, \chi_{2}^{2}=0.39(p=0.82)$

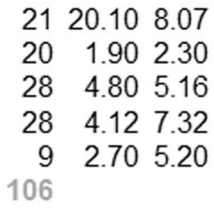

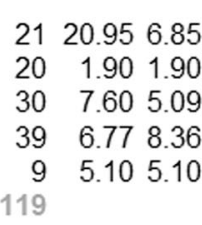

$\begin{array}{rrr}21 & 20.95 & 6.85 \\ 20 & 1.90 & 1.90 \\ 30 & 7.60 & 5.09 \\ 39 & 6.77 & 8.36 \\ 9 & 5.10 & 5.10 \\ 119 & & \end{array}$
$\begin{array}{lll}7 & 0.58 & 0.58\end{array}$
0.400 .19
4.104 .00

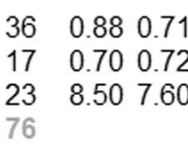

$\begin{array}{lll}36 & 0.88 & 0.71 \\ 17 & 0.70 & 0.72 \\ 23 & 8.50 & 7.60 \\ 76 & & \end{array}$

\section{Standardised Mean \\ Difference}

SMD

$95 \%-\mathrm{Cl}$
Fig. 6 Forest plot of depression outcomes. Footnote: scale by article in alphabetical order. Cohen and Miller 2009: Center for Epidemiological Studies-Depression Scale; Dobie et al. 2016: Depression, Anxiety and Stress Scales (DASS)-Depression; Fortney et al. 2013: DASSDepression; Foureur et al. 2013: DASS-Depression; Pipe et al. 2009:

"asset-based" psychological outcomes (e.g. satisfaction with life), as well as outcomes associated with well-being (e.g. emotional intelligence). Let us consider these classes in turn.

First, the analysis supports the contention that MBIs can be helpful in addressing the mental health needs of HCPs. Effect sizes ranging from small to medium were observed in the expected direction (i.e. reduced burden) for all measures, including anxiety $(-1.01$ for non-randomised trials, -0.49 for RCTs,

\section{Study}

Pre-Post

Krasner et al., 2009

Martin-Asuero \& Garcia-Banda, 2010

Noone \& Hastings, 2010

Rimes \& Wingrove, 2011

Foureur, Besley, Burton, Yu, \& Crisp, 2013

Random effects model

Heterogeneity: $I^{2}=0 \%, \tau^{2}=0, \chi_{4}^{2}=0.22(p=0.99)$

\section{RCT}

Shapiro, Schwartz, \& Bonner, 1998

Shapiro, Astin, Bishop, \& Cordova, 2005

Pipe et al., 2009

de Vibe et al., 2013

Manotas, Segura, Eraso, Oggins, \& McGovern, 2014

Martin-Asuero et al., 2014

McConachie, McKenzie, Morris, \& Walley, 2014

Random effects model

Heterogeneity: $I^{2}=12 \%, \tau^{2}=0.0064, \gamma^{2}=6.78(p=0.34)$

\begin{tabular}{rrr}
\multicolumn{3}{c}{$\begin{array}{c}\text { Experimental / Post } \\
\text { Total Mean }\end{array}$} \\
SD
\end{tabular}

$\begin{array}{rrr}37 & 0.07 & 0.06 \\ 10 & 0.47 & 0.34 \\ 15 & 0.33 & 0.17 \\ 144 & 9.20 & 4.00 \\ 36 & 0.30 & 0.26 \\ 43 & 14.90 & 7.50 \\ 66 & 10.16 & 3.37 \\ 351 & & \end{array}$

Fig. 7 Forest plot of distress outcomes. Footnote: scale by article in alphabetical order. De Vibe et al. 2013: General Health Questionnaire 12 (GHQ-12); Foureur et al. 2013: GHQ-12; Krasner et al. 2009: Profile of Moods States (POMS); Manotas et al. 2014: Brief Symptom Inventory-18 (BSI-18); Martín-Asuero and García-Banda 2010: Positive and Negative and -0.31 for pre-post studies), burnout $(-0.31$ RCTs and 0.51 pre-post), depression $(-0.55$ and -0.29$)$, distress $(-0.61$ and -0.54$)$ and stress $(-0.42$ and -0.58$)$. All random effects models performed on negative outcomes, except anxiety (nonrandomised trials), yielded statistically significant results of around half standardised average difference. These findings somewhat align with previous meta-analyses looking at the impact of mindfulness on such measures in non-clinical
Symptom Checklist 90-R (SCL-90-R)-Depression; Rimes and Wingrove 2011: Hospital Anxiety and Depression Scale-Depression; Shapiro et al. 1998: SCL-90-R-Depression; Song and Lindquist 2015: DASSDepression

\section{Control/Pre Standardised Mean}

SMD

$95 \%-\mathrm{Cl}$
6832.6024 .79

$\begin{array}{lll}29 & 0.68 & 0.40\end{array}$

$\begin{array}{lll}34 & 12.24 & 6.37\end{array}$

$2038.90 \quad 8.80$

$\begin{array}{lll}40 & 3.50 & 3.57\end{array}$

191

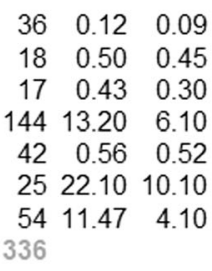
Difference
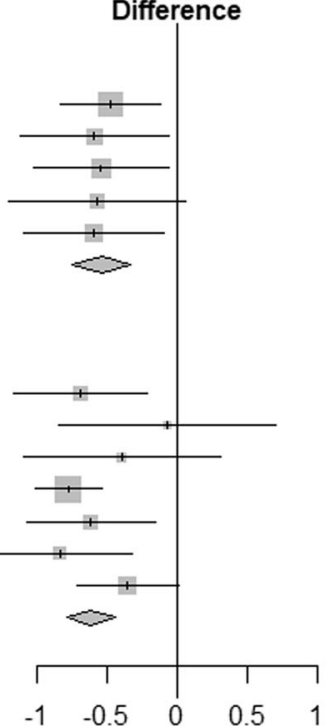

$-0.47[-0.83 ;-0.12]$

$-0.59[-1.12 ;-0.06]$

$-0.54[-1.03 ;-0.06]$

$-0.57[-1.20 ; 0.06]$

$-0.59[-1.09 ;-0.10]$

$-0.54[-0.75 ;-0.33]$
Affect Scales (PANAS)-Negative Affect, Symptom Checklist 90-R (SCL90-R)-GSI; Martín-Asuero et al. 2014: POMS; McConachie et al. 2014: GHQ-12; Noone and Hastings 2010: GHQ-12; 2010 Pipe et al. 2009: SCL90-R-GSI; Rimes and Wingrove 2011: Reflection-Rumination Questionnaire; Shapiro et al. 2005: BSI-18; Shapiro et al. 1998: SCL-90-R-GSI 


\begin{tabular}{|c|c|c|c|}
\hline \multirow{2}{*}{ Study } & \multicolumn{3}{|c|}{ Experimental / Post } \\
\hline & & & \\
\hline \multicolumn{4}{|l|}{ Pre-Post } \\
\hline Cohen \& Miller, 2009 & & 25.86 & 8.97 \\
\hline Aggs, \& Bambling, 2010 & 47 & 2.80 & 1.60 \\
\hline Martin-Asuero \& Garcia-Banda, 2010 & 29 & 0.58 & 0.24 \\
\hline Noone \& Hastings, 2010 & 34 & 60.29 & 19.16 \\
\hline Rimes \& Wingrove, 2011 & 20 & 21.90 & 5.80 \\
\hline Brady, OConnor, Burgermeister, \& Hanson, 2009 & 16 & 39.56 & 20.63 \\
\hline Newsome, Waldo, \& Gruszka, 2012 & 31 & 15.10 & 5.83 \\
\hline Cate, Azocar, \& Kreitzer, 2013 & 36 & 12.39 & 5.71 \\
\hline Bond et al., 2013 & 24 & 1.48 & 2.21 \\
\hline Fortney, Luchterhand, Zakletskaia, et al., 2013 & 28 & 14.10 & 5.25 \\
\hline Besley, Burton, Yu, \& Crisp, 2013 & 28 & 7.28 & 7.13 \\
\hline Hopkins \& Proeve, 2013 & 11 & 18.73 & 5.06 \\
\hline Bonifas \& Napoli, 2014 & 77 & 17.70 & 3.00 \\
\hline Hallman, OConnor, Haseu, \& Brady, 2014 & 12 & 13.67 & 5.45 \\
\hline Dobie, Tucker, Ferrari, \& Rogers, 2015 & 9 & 12.20 & 12.10 \\
\hline Gauthier, Meyer, Grefe, \& Gold, 2015 & 42 & 74.03 & 10.46 \\
\hline Singh et al., 2015 & 9 & 13.00 & 2.74 \\
\hline Pflugeisen, Drummond, Ebersole, et al., 2016 & 19 & 12.30 & 6.80 \\
\hline Random effects model & 493 & & \\
\hline Heterogeneity: $I^{2}=66 \%, \tau^{2}=0.1539, \chi_{17}^{2}=50.5($ & & & \\
\hline
\end{tabular}

RCT

Shapiro, Astin, Bishop, \& Cordova, 2005 de Vibe et al., 2013

Erogul, Singer, Mclntyre, \& Stefanov, 2014

Manotas, Segura, Eraso, Oggins, \& McGovern, 2014

West et al., 2014

Burnett \& Pettijohn, 2015

Random effects model

Heterogeneity: $I^{2}=46 \%, \tau^{2}=0.0487, \chi_{\theta}^{2}=11.04(p=0.09)$
Song \& Lindquist, 2015
Control / Pre Total Mean SD

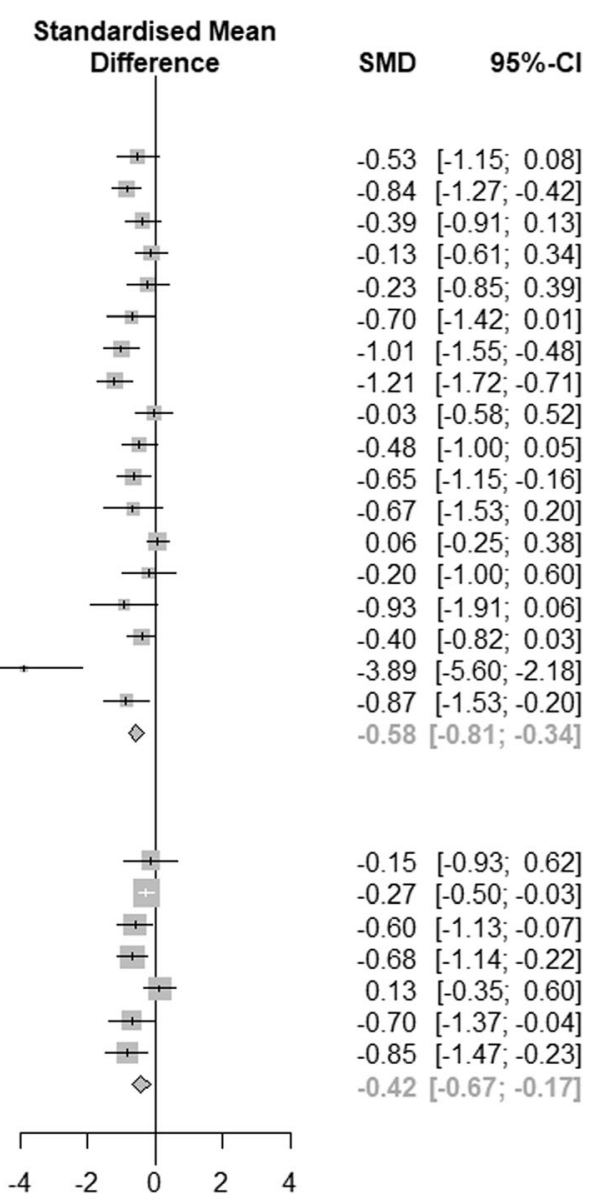

$2130.24 \quad 6.98$

$\begin{array}{lll}47 & 4.41 & 2.15\end{array}$

$\begin{array}{lll}29 & 0.70 & 0.36\end{array}$

3463.1823 .50

$2023.40 \quad 7.00$

1653.6318 .27

$3121.58 \quad 6.75$

$3620.64 \quad 7.61$

$\begin{array}{lll}27 & 1.55 & 2.21\end{array}$

$\begin{array}{lll}30 & 16.60 & 5.11\end{array}$

$\begin{array}{lll}40 & 12.72 \quad 8.89\end{array}$

$\begin{array}{lll}11 & 23.00 & 7.09\end{array}$

$\begin{array}{lll}77 & 17.50 & 3.40\end{array}$

$1214.83 \quad 5.72$

924.7013 .50

$4578.92 \quad 13.71$

$927.00 \quad 4.00$

$1918.40 \quad 7.00$ 513

\begin{tabular}{rrrrrr}
10 & 21.22 & 6.90 & 18 & 22.17 & 5.60 \\
144 & 18.40 & 6.80 & 144 & 20.30 & 7.40 \\
28 & 13.30 & 5.10 & 30 & 17.30 & 7.70 \\
36 & 1.17 & 0.40 & 42 & 1.57 & 0.70 \\
34 & 14.80 & 7.28 & 34 & 13.90 & 6.61 \\
20 & 16.00 & 6.20 & 17 & 20.30 & 5.70 \\
21 & 7.40 & 4.90 & 23 & 13.70 & 8.90 \\
293 & & \multicolumn{4}{c}{308} \\
\end{tabular}

$-0.53 \quad[-1.15 ; 0.08]$

$-0.84 \quad[-1.27 ;-0.42]$

$-0.91,0.13]$

$-0.23 \quad[-0.85 ; 0.39]$

$-0.70 \quad[-1.42 ; 0.01]$

-0.03 [-0.58; 0.52$]$

$-0.48 \quad[-1.00 ; 0.05]$

$0.65[-1.15 ;-0.16]$

$[-1.53 ; 0.20]$

$0.20[-1.00 ; 0.60]$

$-0.93[-1.91 ; 0.06]$

$-0.40 \quad[-0.82 ; 0.03]$

$-0.87[-1.53 ;-0.20]$

$-0.58[-0.81 ;-0.34]$

$-0.15 \quad[-0.93 ; 0.62]$

$-0.27[-0.50 ;-0.03]$

$-0.60[-1.13 ;-0.07]$

$68[-1.14 ;-0.22]$

$-0.85[-1.47 ;-0.23]$

$-0.42[-0.67 ;-0.17]$
Fig. 8 Forest plot of stress outcomes. Footnote: scale by article in alphabetical order. Aggs \& Bambling 2010: Single-Item 10-Point Stress Scale; Bazarko et al. 2013: Perceived Stress Scale (PSS); Bond et al. 2013: PSS; Bonifas and Napoli 2014: PSS; Brady et al. 2012: Mental Health Professionals Stress Scale; Burnett and Pettijohn 2015 (vs passive intervention): PSS; Cohen and Miller 2009: PSS; De Vibe et al. 2013: Perceived Medical School Stress; Dobie et al. 2016: Depression Anxiety Stress Scale (DASS)-Stress; Erogul et al. 2014: PSS; Fortney et al. 2013:

populations (but not HCPs specifically). For instance, analysing 29 studies of MBSR, Khoury et al. (2015) observed a large effect size with respect to stress, a medium effect in relation to anxiety, distress, and depression, and a small effect for burnout. The findings here are promising, given the mental health burdens faced by HCPs, with surveys suggested that mental health issues may be even higher among HCPs than in the general population. For instance, a longitudinal study of 318 GPs by Firth-Cozens (1998) found that $16.8 \%$ were above the threshold for depression on the depression scale of the Symptom Checklist 90, with 9.9\% having some suicidal ideation (4.6\% more than "occasionally"). These figures contrast with estimates that around $2.3 \%$ of the general UK adult population experience a depressive episode at any one time (i.e. in the past week), with $9 \%$ experiencing mixed anxiety and depressive disorder (The Health and Social Care Information Centre 2009). There are many hypothesised reasons for this greater liability to depression among HCPs, including
DASS-Stress; Fortney et al. 2013: PSS; Foureur et al. 2013: DASSStress; Gauthier et al. 2015: Nursing Stress Scale; Hallman et al. 2014: PSS; Hopkins and Proeve 2013: PSS; Manotas et al. 2014: PSS; MartínAsuero and García-Banda 2010: Survey of Recent Life Experiences; Newsome et al. 2012: PSS; Noone and Hastings 2010: Staff Stressor Questionnaire; Pflugeisen et al. 2016: PSS; Rimes and Wingrove 2011: PSS; Shapiro et al. 2005: PSS; Singh et al. 2015: PSS; Song and Lindquist 2015: DASS-Stress; West et al. 2014: PSS

personality traits such as perfectionism, burdens of clinical responsibility, and reluctance to seek treatment (Bright and Krahn 2011). Whatever the reasons, it is encouraging that MBIs appear to help in this regard, reflecting the more established efficacy of MBIs such as MBCT with respect to depression (Segal et al. 2002). In terms of moderator analyses, no statistically significant differences were observed for any negative outcome.

Similarly, the relatively positive results regarding stress are welcome here, especially given that stress appears to be generally higher among HCPs than in the general population. For instance, Firth-Cozens (2003) reported that the proportion of HCPs being above threshold levels of stress is around $28 \%$ in surveys, compared with about $18 \%$ in the general working population. As with depression, a similar range of factors have been implicated in elevated stress levels among HCPs, from long working hours to the burden of clinical responsibility (Sochos et al. 2012). Unfortunately, as highlighted above, these 


\begin{tabular}{|c|c|c|c|c|c|c|}
\hline \multirow[b]{2}{*}{ Study } & \multicolumn{3}{|c|}{ Experimental / Post } & \multicolumn{3}{|c|}{ Control / Pre } \\
\hline & Total & Mean & SD & Total & Mean & \\
\hline \multicolumn{7}{|l|}{ Pre-Post } \\
\hline Rimes \& Wingrove, 2011 & 20 & 20.30 & 2.40 & 20 & 19.00 & \\
\hline Newsome, Waldo, \& Gruszka, 2012 & 31 & 3.52 & 0.64 & 31 & 2.78 & \\
\hline Bazarko, Cate, Azocar, \& Kreitzer, 2013 & 36 & 3.72 & 0.66 & 36 & 2.83 & \\
\hline Bond et al., 2013 & 24 & 3.25 & 2.17 & 27 & 2.88 & \\
\hline Fortney, Luchterhand, Zakletskaia, et al., 2013 & 28 & 27.40 & 4.38 & 30 & 27.60 & \\
\hline Gauthier, Meyer, Grefe, \& Gold, 2015 & 42 & 3.24 & 0.74 & 45 & 3.08 & \\
\hline Raab, Sogge, Parker, \& Flament, 2015 & 22 & 21.37 & 3.93 & 22 & 19.37 & \\
\hline Random effects model & 203 & & & 211 & & \\
\hline
\end{tabular}

Heterogeneity: $I^{2}=71 \%, \tau^{2}=0.1811, \chi_{\theta}^{2}=20.93(p<0.01)$

RCT

Shapiro, Schwartz, \& Bonner, 1998

Shapiro, Astin, Bishop, \& Cordova, 2005

Erogul, Singer, Mclntyre, \& Stefanov, 2014

West et al., 2014

Random effects model

Heterogeneity: $I^{2}=59 \%, \tau^{2}=0.1102, \chi_{3}^{2}=7.32(p=0.06)$

$\begin{array}{rrrrrr}37 & 84.00 & 24.40 & 36 & 73.00 & 21.60 \\ 10 & 20.15 & 4.20 & 18 & 20.07 & 3.40 \\ 28 & 3.60 & 0.50 & 30 & 3.00 & 0.80 \\ 34 & 121.80 & 12.25 & 34 & 122.40 & 13.27 \\ 109 & & & 118 & & \end{array}$

$37 \quad 84.0024 .40$

$3.60 \quad 0.50$ (6)
Fig. 9 Forest plot of compassion outcomes. Footnote: scale by article in alphabetical order. Bazarko et al. 2013: Self-Compassion Scale (SCS); Bond et al. 2013: SCS; Erogul et al. 2014: SCS; Fortney et al. 2013: Santa

burdens have only increased over recent years, due to factors such as curbs on healthcare spending meaning that overwork has become even more acute. As noted above, a survey of National Health Service staff found that $61 \%$ reporting feeling stressed all or most of the time, and 59\% stating that their stress is worse this year than last year (Dudman et al. 2015). Thus, the small to medium effect size observed in relation to stress here is notable, although this was less than the large effect size observed by Khoury et al.'s (2015) aforementioned metaanalysis of MBSR in non-clinical populations (not HCPs specifically). Such findings show that mindfulness may have a useful role to play in ameliorating work-based stress and burnout. However, while these results are encouraging, concerns have been expressed about MBIs being used in occupational contexts as a sticking plaster to merely treat the symptoms of a "toxic" or otherwise challenging work environment, rather than undertaking the more difficult task of creating environments more hospitable to employees (Van Gordon et al. 2016). Moreover, such interventions can potentially place the onus on employees to "cope" with stress and burnout via MBIs, rather than on employers to render the work itself less

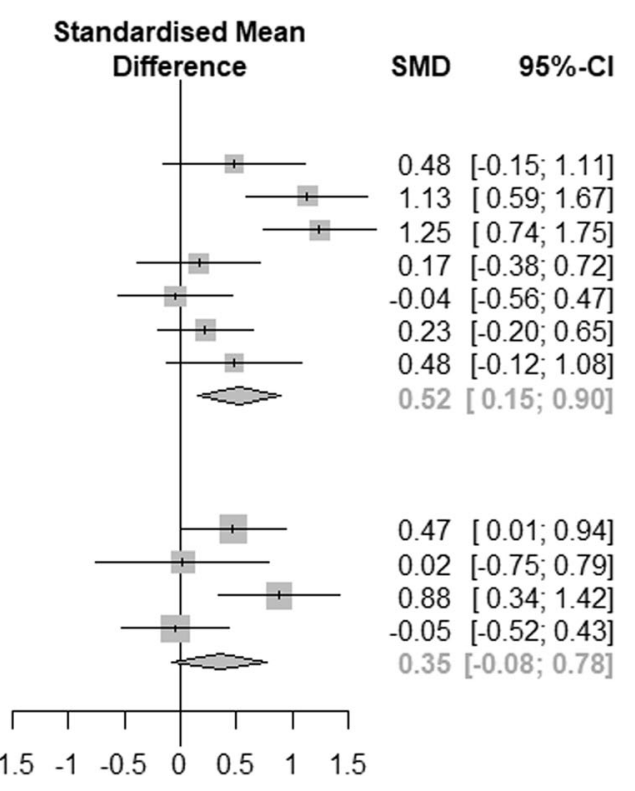

Clara Brief Compassion Scale; Gauthier et al. 2015: SCS; Newsome et al. 2012: SCS; Raab et al. 2015: SCS; Rimes and Wingrove 2011: SCS; Shapiro et al. 2005: SCS

demanding. As such, while MBIs may well be helpful to HCPs in terms of alleviating mental health issues, it is vital that their underlying structural causes are also addressed.

The second class of well-being outcomes are more positive "asset-based" measures. These include outcomes that have recently come to prominence via the burgeoning paradigm of "positive psychology" (Seligman and Csikszentmihalyi 2000), like satisfaction with life (Diener et al. 1985) (even if such topics predate the emergence of positive psychology in the late 1990s). The relative lack of attention to such outcomes in the HCP literature considered here is somewhat reflective of the field of psychology more broadly. That is, one rationale behind the emergence of the positive psychology movement was the charge that mainstream psychology tended to be concerned with disorder, deficit and dysfunction, and paid relatively little attention to "the brighter sides of human nature," as Linley and Joseph (2004, p. 4) put it, to the ways in which humans excel and flourish. One of positive psychology's foundational metaphors of PP was of a continuum, stretching from a nominal minus 10, through zero and up to plus 10 (Keyes 2002). On that metaphor, ameliorating deficits such as mental disorder

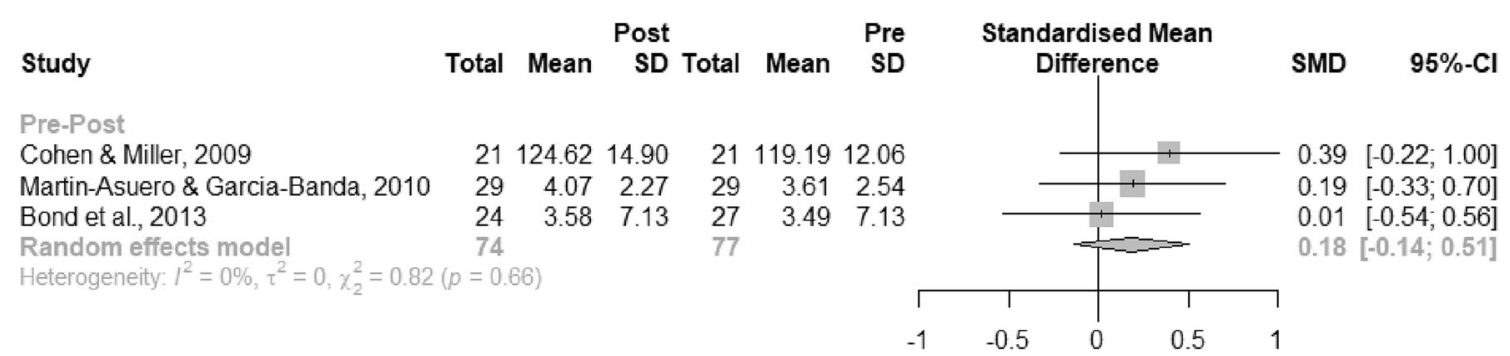

Fig. 10 Forest plot of emotional intelligence outcomes. Footnote: scale by article in alphabetical order. Bond et al. 2013: Self-Regulation Questionnaire; Cohen and Miller 2009: Self-Report of Emotional Intelligence; Martín-Asuero and García-Banda 2010: Emotional Control Questionnaire 


\begin{tabular}{lrrr} 
& \multicolumn{3}{c}{$\begin{array}{c}\text { Experimental / Post } \\
\text { Total }\end{array}$} \\
& & & \\
Study & 59 & 120.60 & 9.21 \\
Pre-Post & 20 & 20.77 & 2.47 \\
Krasner et al., 2009 & 36 & 123.59 & 9.31 \\
Rimes \& Wingrove, 2011 & 24 & 5.80 & 1.78 \\
Bazarko, Cate, Azocar, \& Kreitzer, 2013 & 11 & 17.73 & 2.72 \\
Bond et al., 2013 & 150 & & \\
Hopkins \& Proeve, 2013 & & \\
Random effects model & &
\end{tabular}

Fig. 11 Forest plot of empathy outcomes. Footnote: scale by article in alphabetical order. Bazarko et al. 2013: Jefferson Scale of Physician Empathy (JSPE); Bond et al. 2013: JSPE; Hopkins and Proeve 2013:

constitutes bringing people up to "zero." That is hugely beneficial, as far as it goes. But being at "zero" does not necessarily mean people are flourishing (e.g. truly thriving, and fulfilling their potential). Thus, positive psychology sought to draw attention to outcomes that might represent the positive integers in this metaphor. The current review sought to capture this aspect of well-being, including such outcomes as satisfaction with life (e.g. Cohen and Miller 2009). Overall a small to medium effect size was observed $(0.27$ for RCTs and 0.49 for pre-post), which is encouraging (with no significant results observed for any moderator). However, this is a relatively understudied domain of well-being in the literature on mindfulness in HCPs, and further research is needed.

Relatedly, the review also included "positive" outcomes measures that, although not constitutive of well-being in themselves, are closely related to it. These include

\section{Control/Pre}

Mean SD

$68 \quad 117.209 .50$

$\begin{array}{lr}20 & 20.302 .83\end{array}$

36116.598 .98

$27 \quad 5.641 .78$

18.452 .65

162

(1)

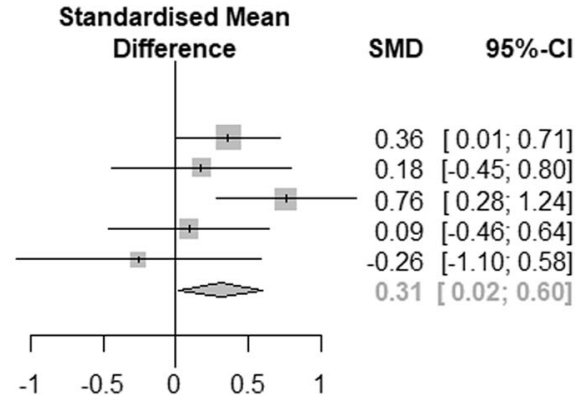

Interpersonal Reactivity Index (IRI); Krasner et al. 2009: JSPE; Rimes and Wingrove 2011: IRI

mindfulness itself, for which an effect size of 0.52 was observed for pre-post studies, although only 0.34 for RCTs (which moreover was non-significant). The latter result is somewhat surprising and suggests that whatever benefits participants may be gaining from MBIs, it is unclear the extent to which this is attributable to increases in mindfulness itself (since, after all, this did not increase significantly in RCTs), as opposed to accruing from other rewarding components of the programme (e.g. a supportive social environment). Mindfulness also yielded some interesting results in terms of subgroup and meta-regression analyses, with variability with respect to the type of intervention (with greater effect sizes in mindfulness among studies that did not use the MBSR programme).

Other positive outcomes of note included empathy and compassion. In this respect though, while significant effect Experimental / Post Control / Pre
Total Mean SD Total Mean SD

\section{Study}

Pre-Post

Cohen \& Miller, 2009

Fortney, Luchterhand, Zakletskaia, et al., 2013

Foureur, Besley, Burton, Yu, \& Crisp, 2013

Bonifas \& Napoli, 2014

Raab, Sogge, Parker, \& Flament, 2015

Random effects model

Heterogeneity: $I^{2}=58 \%, \tau^{2}=0.0877, \chi_{4}^{2}=9.59(p=0.05)$

RCT

Shapiro, Schwartz, \& Bonner, 1998

Shapiro, Astin, Bishop, \& Cordova, 2005

Mackenzie, Poulin, \& Seidman-Carlson, 2006

de Vibe et al., 2013

Erogul, Singer, McIntyre, \& Stefanov, 2014

McConachie, McKenzie, Morris, \& Walley, 2014

West et al., 2014

Random effects mode!

Heterogeneity: $I^{2}=0 \%, \tau^{2}=0, x_{\theta}^{2}=5.31(p=0.50)$

$\begin{array}{rrr}37 & 2.80 & 0.57 \\ 10 & 24.80 & 6.70 \\ 16 & 46.78 & 4.87 \\ 144 & 6.80 & 1.40 \\ 28 & 80.50 & 10.00 \\ 66 & 50.91 & 5.98 \\ 34 & 4.00 & 0.65 \\ 335 & & \end{array}$

335

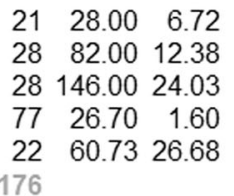

776

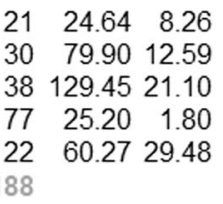

$\begin{array}{rrr}36 & 2.60 & 0.68 \\ 18 & 23.83 & 6.20 \\ 14 & 46.08 & 5.34 \\ 144 & 6.10 & 1.80 \\ 30 & 77.10 & 14.10 \\ 54 & 49.88 & 6.29 \\ 34 & 4.10 & 0.75 \\ 330 & & \end{array}$

Fig. 12 Forest plot of life satisfaction \& flourishing outcomes. Footnote: scale by article in alphabetical order. Bonifas and Napoli 2014: Quality of Life Index; Cohen and Miller 2009: Meaning in Life Questionnaire (MLQ)-Search for Meaning, MLQ-Presence of meaning, Satisfaction With Life Scale (SWLS); De Vibe et al. 2013: Subjective Wellbeing Scale; Erogul et al. 2014: Resilience Scale; Foureur et al. 2013: Sense

\section{Mean SD}

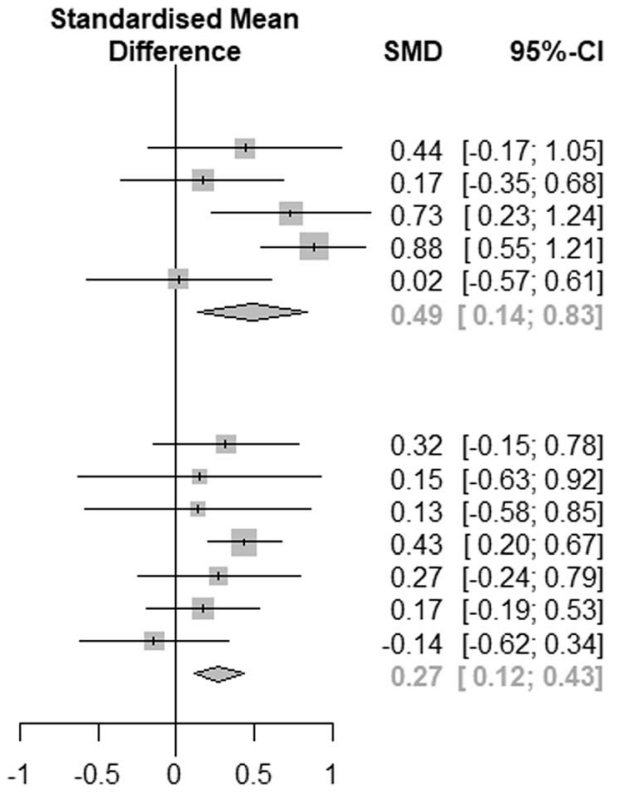

of Coherence (SOC); Mackenzie et al. 2006: SOC, Smith Relaxation Dispositions Inventory, SWLS, Job Satisfaction Scale; McConachie et al. 2014: Warwick-Edinburgh Mental Well-Being Scale; Raab et al. 2015: Quality of life inventory; De Vibe et al. 2013: Subjective Wellbeing Scale; Shapiro et al. 2005: SWLS; Shapiro et al. 1998: Index of Core Spiritual Experiences; West et al. 2014: Physician Job Satisfaction Scale 


$\begin{array}{ll}\text { Experimental/Post } & \text { Control/Pre } \\ \text { Study } & \text { Total Mean SD Total Mean SD }\end{array}$

Pre-Post

Cohen \& Miller, 2009

Krasner et al., 2009

Aggs, \& Bambling, 2010

Rimes \& Wingrove, 2011

Brady, OConnor, Burgermeister, \& Hanson, 2009

Newsome, Waldo, \& Gruszka, 2012

Hopkins \& Proeve, 2013

Hallman, OConnor, Haseu, \& Brady, 2014

Dobie, Tucker, Ferrari, \& Rogers, 2015

Gauthier, Meyer, Grefe, \& Gold, 2015

Kemper \& Khirallah, 2015

Pflugeisen, Drummond, Ebersole, et al., 2016

Random effects model

Heterogeneity: $I^{2}=46 \%, \tau^{2}=0.0562, \chi_{11}^{2}=20.29(p=0.04)$

RCT

Mackenzie, Poulin, \& Seidman-Carlson, 2006

de Vibe et al., 2014

Manotas, Segura, Eraso, Oggins, \& McGovern, 2014

Martin-Asuero et al., 2014

Song \& Lindquist, 2015

Random effects model

Heterogeneity: $I^{2}=72 \%, \tau^{2}=0.1362, \chi_{4}^{2}=14.3(p<0.01)$

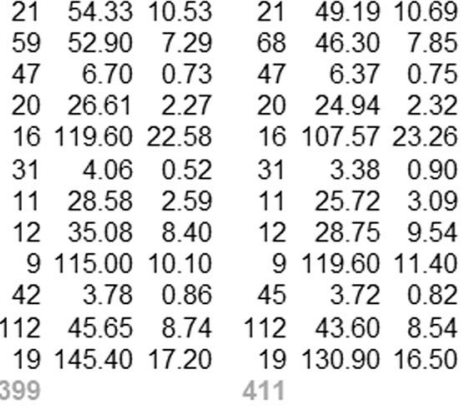
399

$\begin{array}{rrrrrr}16 & 43.56 & 9.29 & 14 & 41.00 & 11.89 \\ 144 & 25.94 & 2.56 & 144 & 25.60 & 2.75 \\ 38 & 3.40 & 0.32 & 42 & 3.38 & 0.37 \\ 43 & 141.60 & 16.50 & 25 & 121.60 & 16.00 \\ 21 & 80.60 & 11.30 & 23 & 79.00 & 12.60 \\ 262 & & & 248 & & \end{array}$

Fig. 13 Forest plot of mindfulness and awareness outcomes. Footnote: scale by article in alphabetical order. Aggs \& Bambling 2010: Five Facet Mindfulness Questionnaire (FFMQ); Brady et al. 2012: Sense of Self Scale, Toronto Mindfulness Scale (TMS); Cohen and Miller 2009: Mindful Attention Awareness Scale (MAAS); de Vibe et al. 2013: FFMQ; Dobie et al. 2016: Kentucky Inventory of Mindfulness Skills (KIMS); Gauthier et al. 2015: MAAS; Hallman et al. 2014: TMS; Hopkins and Proeve 2013:

sizes were observed in pre-post studies for both empathy (0.31) and compassion (0.52), the compassion effect size (0.35) in RCTs was non-significant (while RCT calculations were not possible for empathy due to insufficient studies). Also of note here is the moderating factor of MBI, wherecontrary to the mindfulness outcomes reported abovehigher effect sizes were observed in studies that did use the original MBSR protocol. These conflicting findings regarding moderator variables preclude us from making any simple generalisations about which type of MBI is most effective. More generally, qualities of empathy and compassion are not only relevant in a HCP context because of their close association with well-being, such as the possibility that they provide a buffer against stress (Cosley et al. 2010). There is a significant literature though on the risks of "compassion fatigue" among HCPs (Coetzee and Klopper 2010), which emphasises the importance, among other things, of HCPs developing self-compassion (Boellinghaus et al. 2014).) Empathy and compassion are further interesting here, since in a healthcare context, these qualities are regarded as important occupational skills, for instance being linked to better outcomes for patients (Mannion 2014). This finding aligns with reviews which have reported on job performance metrics in HCPs, such as Guillaumie et al. (2017), who observed - in relation to mindfulness-improved

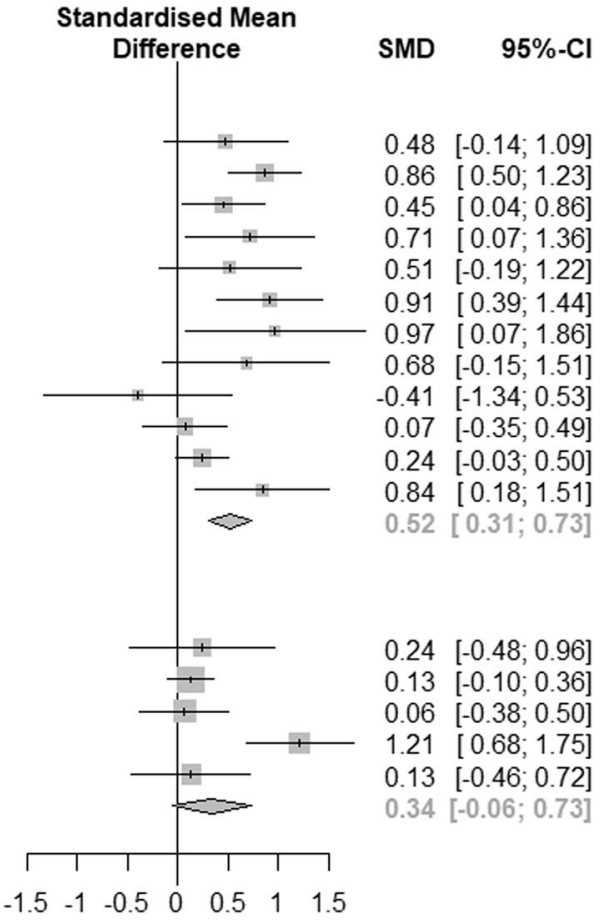

FFMQ; Kemper and Khirallah 2015: Cognitive and Affective Mindfulness Scale-Revised, MAAS; Krasner et al. 2009: Baer Mindfulness Scale; Mackenzie et al. 2006: Smith Relaxation Dispositions Inventory; Manotas et al. 2014: FFMQ; Martín-Asuero et al. 2014: FFMQ; Newsome et al. 2012: MAAS; Pflugeisen et al. 2016: KIMS; Rimes and Wingrove 2011: FFMQ; Song and Lindquist 2015: MAAS

communication with colleagues, greater sensitivity to patients' experiences, clearer analyses of complex situations, and emotional regulation in stressful contexts, and likewise McConville et al. (2017), who observed better learning and clinical performance among health professional students.

This class of positive well-being-related outcomes here also included emotional intelligence and regulation. The interest in such outcomes lies, in part, with the possibility that they may play mediating roles with respect to the outcomes considered above. For instance, emotional intelligence and regulation have been studied as coping resources that can mitigate the deleterious impact of work demands for HCPs (Weng et al. 2011). These outcomes are also relevant, since theoretically they represent one of the strongest candidates for the way in which mindfulness might exert its beneficial effects upon all the outcomes considered in this review. As outlined in the introduction, theorists such as Shapiro et al. (2006) have proposed that a key way in which mindfulness operates beneficially is through a process of "reperceiving," whereby people are empowered to "decentre" from distressing qualia that might otherwise generate distress, etc. And, reperceiving could be regarded as one facet of a more general capacity of emotion regulation. For instance, Walsh and Shapiro (2006) define meditation as "a family of self-regulation practices that focus on training attention and awareness in order to bring 
mental processes under greater voluntary control and thereby foster general mental well-being" (pp. 228-229). However, although improvements were noted here with respect to emotional intelligence/regulation, surprisingly (given the abovementioned theoretical background), the effects did not reach statistical significance. Clearly, this makes one wary here about definitively granting these outcomes a pivotal role in mediating the effects of MBIs on the outcomes above and highlights the need for further research on the relevance of these psychological processes to whatever benefits may be conferred by mindfulness practice.

Overall, though, the results are fairly encouraging in terms of the value of MBIs for HCPs. However, there are various issues with the research base which must temper one's enthusiasm here and which limit the conclusions that can be drawn. The quality assessment revealed considerable variation among studies, with several prominent issues. The first is that older studies tended not to use an RCT design and more generally had a poorer quality of design compared to more recent studies. A second issue is that studies overwhelmingly featured a majority of female participants; this raises doubts concerning the ecological validity of these studies when it comes to their relevance for both males and females (and see Lomas et al. (2015a) for potential gendered differences in the way men may respond to meditation practice). A third issue was blinding, i.e. whether or not participants were aware of the research question and whether assessors were aware of the intervention, which was rarely addressed by studies.

Furthermore, there are other issues beyond those around quality. First, there is considerable heterogeneity in the design of the studies-including type of MBI and outcome measures - which makes it difficult to conduct comparative assessments and hence to draw robust conclusions about the research as a whole. A further issue is that the research is biased towards "negative" psychiatric outcomes (e.g. anxiety, stress, depression), with relatively little attention to "positive" outcomes that are specifically relevant to the work arena, such as work engagement or creativity. Finally, despite not having obtained statistically significant results in our calculations, our appraisal of the literature base is likely to have been hindered by publication bias, i.e. the "file-drawer problem," in that studies with less conclusive or even negative results are less likely to be published (Smith 1980). It was not feasible to collect data from unpublished trials of MBIs with HCPs, which means that the studies reviewed must inevitably be regarded as a somewhat selective survey of the studies that have been conducted in this arena. As an additional point, it should also be noted that it was necessary to perform the calculations with moderating variables using all study designs together (rather than separately according to specific designs, i.e. randomised vs non-randomised). The reason is that separating such analyses by specific designs would generate an unwieldy proliferation of subgroups, many of which would have had just one or even no studies within them. Future meta-analyses, with a greater pool of studies to draw on, may well be able to perform calculations separated by study designs, which would be ideal.

Based on the critiques above, the following recommendations can be made vis-à-vis future work in this area, including in relation to the (a) outcomes, (b) study design, (c) type of MBI, and (d) cost-benefit analyses. First, it would be good to see a diversification of outcome measures. Currently, most studies focus on deficit-based well-being measures, such as anxiety and stress. While those outcomes are important, and the focus on them is understandable given the clinical context in which MBIs were developed, they do not provide the "whole picture" with regard to well-being. As fields like positive psychology have emphasised, well-being is also a question of asset-based outcomes (whose presence is indicative of well-being), such as life satisfaction or positive affect. As such, we recommend that all studies consider including at least one such asset-based outcome in their assessment. Relatedly, when researching MBIs in occupational contexts specifically, we also recommend the inclusion of asset-based outcomes that are particularly germane to this arena, but which have so far received hardly attention at all (and none in the studies reviewed here). These could include, for instance, creativity and leadership (see Kudesia (2015) and George (2012) for reflections on links in the workplace between mindfulness and creativity and leadership respectively).

Second, our QATQS review of the general quality of studies leads us to several recommendations regarding the design of the research. Most importantly, where possible, studies should implement an RCT design, ideally with large numbers of participants (determined by a priori power calculations drawing on estimated effect size). Moreover, in addition to a wait-list control protocol, the design of studies would be improved if trials included an "active" control group. A good example of this in an occupational context is Wolever et al. (2012), who included yoga as an active control. Such designs will better enable any positive effects to be ascribed to mindfulness per se (i.e. rather than simply being involved in an absorbing group activity). Relatedly, studies should pay more attention to the extent to which participants are actually practising mindfulness (e.g. in terms of adherence to homework activities). As Vettese et al. (2009) noted, failure to track such participation is a perennial issue in MBI research, and this trend was observed in the studies analysed here. Additionally, beyond people simply participating in an MBI, much more knowledge is needed about the extent and quality of their involvement with meditation. In that respect, besides quantitatively tracking participation, studies could incorporate a qualitative element to their assessment (see Lomas et al. (2013, 2014a, b, 2015a, 2016) on the value of qualitative analyses in relation to mindfulness practice). 
Third, where possible, trials should involve well-established MBIs (i.e. rather than bespoke adaptations), to better enable comparison and aggregation across studies. Of the 81 studies analysed in Lomas et al.'s (2018) general systematic review of HCPs - of which the current paper provides a meta-analysis of 42 - the 56 intervention studies used a range of different MBIs. These included MBSR $(n=9)$, MBSR adaptations (15), and MBCT (5), together with a range of other less-well-established programmes (16), as well as bespoke interventions seemingly created for that particular study (21). For the purposes of assessing the value of MBIs in occupational contexts it would be helpful - at least in this point in our early understanding of this particular context - for studies to use established MBIs such as MBSR and MBCT, rather than creating bespoke programmes or adaptations. Having said that though, we also recognise the value of moving beyond MBIs developed primarily for clinical contexts (e.g. MBSR), and creating MBIs specifically for the workplace, including for particular types of occupation (e.g. HCPs). For instance, Goodman and Schorling (2012) created and used a bespoke MBSR adaptation called "Mindfulness for Healthcare Providers," which was specifically tailored for a HCP context. As such, we would not want to discourage that kind of innovation. Thus, as the research moves forward, it will be helpful to see a balance between the implementation and assessment of established MBIs on the one hand and innovation and adaption of these into occupational contexts on the other.

Finally, the case for implementing mindfulness in occupational contexts will be enhanced considerably - certainly from the perspective of employers - through cost-benefit analyses. If MBIs can be seen to generate an overall net gain, there are strong incentives for these to be introduced in the workplace. Unfortunately though, few such analyses currently exist (Edwards et al. 2015). There are some valuable and instructive exceptions though. For instance, analysing the impact of "mindful organising" across three large hospitals, Vogus et al. (2014) calculated that this generated a $13.6 \%$ decrease in turnover, representing an average hospital saving of between $\$ 169,000$ and $\$ 1,014,560$. Such analyses will be very valuable in terms of generating organisational buy-in to the potential of mindfulness, thus helping facilitate research going forward that can enable the promise of the research reviewed here to be substantiated (see Edwards et al. (2015) for recommendations on conducting such analyses). Nevertheless, despite the limitations and issues with the current research base, the evidence of the value of mindfulness for HCPs is strong, and one might speculate that this will only strengthen over the years ahead.

\section{Compliance with Ethical Standards}

This article does not contain any studies with human participants or animals performed by any of the authors.
Publisher's Note Springer Nature remains neutral with regard to jurisdictional claims in published maps and institutional affiliations.

\section{References}

(All meta-analysis papers are indicated by *)

*Aggs, C., \& Bambling, M. (2010). Teaching mindfulness to psychotherapists in clinical practice: The mindful therapy programme. Counselling and Psychotherapy Research, 10(4), 278-286. https:// doi.org/10.1080/14733145.2010.485690.

Aldao, A., Nolen-Hoeksema, S., \& Schweizer, S. (2010). Emotionregulation strategies across psychopathology: a meta-analytic review. Clinical Psychology Review, 30(2), 217-237. https://doi.org/ 10.1016/j.cpr.2009.11.004.

*Barbosa, P., Raymond, G., Zlotnick, C., Wilk, J., Toomey, I. R., \& Mitchell, I. J. (2013). Mindfulness-based stress reduction training is associated with greater empathy and reduced anxiety for graduate healthcare students. Education for Health, 26(1), 9-14. https://doi. org/10.4103/1357-6283.112794.

*Bazarko, D., Cate, R. A., Azocar, F., \& Kreitzer, M. J. (2013). The impact of an innovative mindfulness-based stress reduction program on the health and well-being of nurses employed in a corporate setting. Journal of Workplace Behavioral Health, 28(2), 107-133.

Begg, C. B., \& Mazumdar, M. (1994). Operating characteristics of a rank correlation test for publication bias. Biometrics, 50(4), 1088-1101. https://doi.org/10.2307/2533446.

Boellinghaus, I., Jones, F. W., \& Hutton, J. (2014). The role of mindfulness and loving-kindness meditation in cultivating self-compassion and other-focused concern in health care professionals. Mindfulness, 5(2), 129-138.

*Bond, A. R., Mason, H. F., Lemaster, C. M., Shaw, S. W., Mullin, C. S., Holick, E. A., \& Saper, R. B. (2013). Embodied health: the effects of a mind-body course for medical students. Medical Education Online, 18(1). https://doi.org/10.3402/meo.v18i0.20699.

*Bonifas, R. P., \& Napoli, M. (2014). Mindfully increasing quality of life: a promising curriculum for MSW students. Social Work Education, 33(4), 469-484. https://doi.org/10.1080/02615479.2013.838215.

Borenstein, M., Hedges, L. V., Higgins, J. P. T., \& Rothstein, H. R. (2009). Multiple outcomes or time-points within a study. In Introduction to meta-analysis (pp. 225-238). Chichester: John Wiley \& Sons, Ltd.

Bourdieu, P. (1986). The forms of capital. In J. G. Richardson (Ed.), Handbook of theory and research for the sociology of education (pp. 241-258). New York: Greenwood.

*Brady, S., O’Connor, N., Burgermeister, D., \& Hanson, P. (2012). The impact of mindfulness meditation in promoting a culture of safety on an acute psychiatric unit. Perspectives in Psychiatric Care, 48(3), 129-137. https://doi.org/10.1111/j.1744-6163.2011.00315.x.

Bright, R. P., \& Krahn, L. (2011). Depression and suicide among physicians. Current Psychiatry, 10(4), 16.

Bühlmayer, L., Birrer, D., Röthlin, P., Faude, O., \& Donath, L. (2017). Effects of mindfulness practice on performance-relevant parameters and performance outcomes in sports: a meta-analytical review. Sports Medicine, 47(11), 2309-2321.

*Burnett, M., \& Pettijohn, C. (2015). Investigating the efficacy of mind-body therapies and emotional intelligence on worker stress in an organizational setting: an experimental approach. Journal of Organizational Culture, Communications and Conflict, 19(1), 146-158.

Burton, A., Burgess, C., Dean, S., Koutsopoulou, G. Z., \& Hugh-Jones, S. (2017). How effective are mindfulness-based interventions for 
reducing stress among healthcare professionals? A systematic review and meta-analysis. Stress and Health, 33(1), 3-13. https:// doi.org/10.1002/smi.2673.

Chambers, R., Gullone, E., \& Allen, N. B. (2009). Mindful emotion regulation: an integrative review. Clinical Psychology Review, 29(6), 560-572. https://doi.org/10.1016/j.cpr.2009.06.005.

Coetzee, S. K., \& Klopper, H. C. (2010). Compassion fatigue within nursing practice: a concept analysis. Nursing \& Health Sciences, 12(2), 235-243.

*Cohen, J. S., \& Miller, L. J. (2009). Interpersonal mindfulness training for well-being: a pilot study with psychology graduate students. Teachers College Record, 111(12), 2760-2774.

Cosley, B. J., McCoy, S. K., Saslow, L. R., \& Epel, E. S. (2010). Is compassion for others stress buffering? Consequences of compassion and social support for physiological reactivity to stress. Journal of Experimental Social Psychology, 46(5), 816-823. https://doi.org/ 10.1016/j.jesp.2010.04.008.

Daya, Z., \& Hearn, J. H. (2017). Mindfulness interventions in medical education: a systematic review of their impact on medical student stress, depression, fatigue and burnout. Medical Teacher, 1-8. https://doi.org/10.1080/0142159X.2017.1394999.

de Chavez, A. C., Backett-Milburn, K., Parry, O., \& Platt, S. (2005). Understanding and researching wellbeing: Its usage in different disciplines and potential for health research and health promotion. Health Education Journal, 64(1), 70-87. https://doi.org/10.1177/ 001789690506400108

Diener, E. (2000). Subjective well-being: the science of happiness and a proposal for a national index. American Psychologist, 55(1), $34-43$. https://doi.org/10.1037/0003-066X.55.1.34.

Diener, E., Emmons, R. A., Larsen, R. J., \& Griffin, S. (1985). The satisfaction with life scale. Journal of Personality Assessment, 49(1), 71-75. https://doi.org/10.1207/s15327752jpa4901_13.

*De Vibe, M., Solhaug, I., Tyssen, R., Friborg, O., Rosenvinge, J. H., Sørlie, T., \& Bjørndal, A. (2013). Mindfulness training for stress management: a randomised controlled study of medical and psychology students. BMC Medical Education, 13(1), 107. https://doi. org/10.1186/1472-6920-13-107.

*Dobie, A., Tucker, A., Ferrari, M., \& Rogers, J. M. (2016). Preliminary evaluation of a brief mindfulness-based stress reduction intervention for mental health professionals. Australasian Psychiatry, 24(1), 42 45. https://doi.org/10.1177/1039856215618524.

Dudman, J., Isaac, A., \& Johnson, S. (2015, 10 June 2015). Revealed: how the stress of working in public services is taking its toll on staff, The Guardian. Retrieved from http://www.theguardian.com/society/ 2015/jun/10/stress-working-public-services-survey.

Eby, L. T., Allen, T. D., Conley, K. M., Williamson, R. L., Henderson, T. G., \& Mancini, V. S. (2017). Mindfulnessbased training interventions for employees: a qualitative review of the literature. Human Resource Management Review. https:// doi.org/10.1016/j.hrmr.2017.03.004

Edwards, R. T., Bryning, L., \& Crane, R. (2015). Design of economic evaluations of mindfulness-based interventions: ten methodological questions of which to be mindful. Mindfulness, 6(3), 490-500.

Emerson, L.-M., Leyland, A., Hudson, K., Rowse, G., Hanley, P., \& Hugh-Jones, S. (2017). Teaching mindfulness to teachers: a systematic review and narrative synthesis. Mindfulness, 8(5), 1136-1149. https://doi.org/10.1007/s12671-017-0691-4.

*Erogul, M., Singer, G., McIntyre, T., \& Stefanov, D. G. (2014). Abridged mindfulness intervention to support wellness in first-year medical students. Teaching and Learning in Medicine, 26(4), 350 356. https://doi.org/10.1080/10401334.2014.945025.

Firth-Cozens, J. (1998). Individual and organizational predictors of depression in general practitioners. British Journal of General Practice, 48(435), 1647-1651.
Firth-Cozens, J. (2003). Doctors, their wellbeing, and their stress: it's time to be proactive about stress - and prevent it. BMJ: British Medical Journal, 326(7391), 670-671.

*Fortney, L., Luchterhand, C., Zakletskaia, L., Zgierska, A., \& Rakel, D. (2013). Abbreviated mindfulness intervention for job satisfaction, quality of life, and compassion in primary care clinicians: a pilot study. Annals of Family Medicine, 11(5), 412-420. https://doi.org/ 10.1370/afm. 1511

*Foureur, M., Besley, K., Burton, G., Yu, N., \& Crisp, J. (2013). Enhancing the resilience of nurses and midwives: pilot of a mindfulness-based program for increased health, sense of coherence and decreased depression, anxiety and stress. Contemporary Nurse, 45(1), 114-125.

Fresco, D. M., Moore, M. T., van Dulmen, M. H. M., Segal, Z. V., Ma, S. H., Teasdale, J. D., \& Williams, J. M. G. (2007). Initial psychometric properties of the experiences questionnaire: validation of a selfreport measure of decentering. Behavior Therapy, 38(3), 234-246. https://doi.org/10.1016/j.beth.2006.08.003.

Gao, Y.-Q., Pan, B.-C., Sun, W., Wu, H., Wang, J.-N., \& Wang, L. (2012). Anxiety symptoms among Chinese nurses and the associated factors: a cross sectional study. BMC Psychiatry, 12(1), 141.

*Gauthier, T., Meyer, R. M. L., Grefe, D., \& Gold, J. I. (2015). An on-thejob mindfulness-based intervention for pediatric ICU nurses: a pilot. Journal of Pediatric Nursing, 30(2), 402-409. https://doi.org/10. 1016/j.pedn.2014.10.005.

George, B. (2012). Mindfulness helps you become a better leader. Harvard Business Review, 26, 21-32.

Givens, J. L., \& Tjia, J. (2002). Depressed medical students' use of mental health services and barriers to use. Academic Medicine, 77(9), 918-921.

*Gockel, A., Burton, D., James, S., \& Bryer, E. (2013). Introducing Mindfulness as a Self-Care and Clinical Training Strategy for Beginning Social Work Students. Mindfulness, 4(4), 343-353. https://doi.org/10.1007/s12671-012-0134-1.

*Goodman, M. J., \& Schorling, J. B. (2012). A mindfulness course decreases burnout and improves well-being among healthcare providers. International Journal of Psychiatry in Medicine, 43(2), 119-128. https://doi.org/10.2190/PM.43.2.b.

*Grepmair, L., Mitterlehner, F., Loew, T., \& Nickel, M. (2007). Promotion of mindfulness in psychotherapists in training: preliminary study. European Psychiatry, 22(8), 485-489. https://doi.org/10. 1016/j.eurpsy.2007.02.004.

Guillaumie, L., Boiral, O., \& Champagne, J. (2017). A mixedmethods systematic review of the effects of mindfulness on nurses. Journal of Advanced Nursing, 73(5), 1017-1034. https://doi.org/10.1111/jan.13176.

*Hallman, I. S., O'Connor, N., Hasenau, S., \& Brady, S. (2014). Improving the culture of safety on a high-acuity inpatient child/ adolescent psychiatric unit by mindfulness-based stress reduction training of staff. Journal of Child and Adolescent Psychiatric Nursing, 27(4), 183-189. https://doi.org/10.1111/jcap.12091.

Higgins, J. P. T., Thompson, S. G., Deeks, J. J., \& Altman, D. G. (2003). Measuring inconsistency in meta-analyses. BMJ, 327(7414), 557560. https://doi.org/10.1136/bmj.327.7414.557.

Higgins, J. P., \& Green, S. (Eds.). (2011). Cochrane handbook for systematic reviews of interventions (Vol. 5). Chichester: WileyBlackwell.

*Hopkins, A., \& Proeve, M. (2013). Teaching mindfulness-based cognitive therapy to trainee psychologists: qualitative and quantitative effects. Counselling Psychology Quarterly, 26(2), 115-130. https:// doi.org/10.1080/09515070.2013.792998.

Hwang, Y.-S., Bartlett, B., Greben, M., \& Hand, K. (2017). A systematic review of mindfulness interventions for in-service teachers: a tool to enhance teacher wellbeing and performance. Teaching and Teacher Education, 64(Supplement C), 26-42. https://doi.org/10.1016/j.tate. 2017.01.015. 
*Johnson, J. R., Emmons, H. C., Rivard, R. L., Griffin, K. H., \& Dusek, J. A. (2015). Resilience training: A pilot study of a mindfulness-based program with depressed healthcare professionals. EXPLORE: The Journal of Science and Healing, 11(6), 433-444. https://doi.org/10. 1016/j.explore.2015.08.002.

Kabat-Zinn, J. (1982). An outpatient program in behavioral medicine for chronic pain patients based on the practice of mindfulness meditation: theoretical considerations and preliminary results. General Hospital Psychiatry, 4(1), 33-47. https://doi.org/10.1016/01638343(82)90026-3.

Kabat-Zinn, J. (2003). Mindfulness-based interventions in context: past, present, and future. Clinical Psychology: Science and Practice, 10(2), 144-156. https://doi.org/10.1093/clipsy.bpg016.

*Kemper, K. J., \& Khirallah, M. (2015). Acute effects of online mindbody skills training on resilience, mindfulness, and empathy. Journal of Evidence-Based Complementary and Alternative Medicine, 20(4), 247-253. https://doi.org/10.1177/ 2156587215575816.

Keyes, C. L. M. (2002). The mental health continuum: from languishing to flourishing in life. Journal of Health and Social Behavior, 43(2), 207-222. https://doi.org/10.2307/3090197.

Khoury, B., Lecomte, T., Fortin, G., Masse, M., Therien, P., Bouchard, V., ... Hofmann, S. G. (2013). Mindfulness-based therapy: a comprehensive meta-analysis. Clinical Psychology Review, 33(6), 763-771. https://doi.org/10.1016/j.cpr.2013.05.005.

Khoury, B., Sharma, M., Rush, S. E., \& Fournier, C. (2015). Mindfulness-based stress reduction for healthy individuals: a metaanalysis. Journal of Psychosomatic Research, 78(6), 519-528. https://doi.org/10.1016/j.jpsychores.2015.03.009.

*Krasner, M. S., Epstein, R. M., Beckman, H., Suchman, A. L., Chapman, B., Mooney, C. J., \& Quill, T. E. (2009). Association of an educational program in mindful communication with burnout, empathy, and attitudes among primary care physicians. JAMA Journal of the American Medical Association, 302(12), 12841293. https://doi.org/10.1001/jama.2009.1384.

Kudesia, R. S. (2015). Mindfulness and creativity in the workplace. In J. Reb \& P. W. B. Atkins (Eds.), Mindfulness in organizations: foundations, research, and applications (pp. 190-212). Cambridge: Cambridge University Press.

Lamothe, M., Rondeau, É., Malboeuf-Hurtubise, C., Duval, M., \& Sultan, S. (2016). Outcomes of MBSR or MBSR-based interventions in health care providers: a systematic review with a focus on empathy and emotional competencies. Complementary Therapies in Medicine, 24, 19-28.

Larson, J. S. (1999). The conceptualization of health. Medical Care Research and Review, 56(2), 123-136. https://doi.org/10.1177/ 107755879905600201

Linley, P. A., \& Joseph, S. (2004). Applied positive psychology: a new perspective for professional practice. In P. A. Linley \& S. Joseph (Eds.), Positive psychology in practice (pp. 3-12). Hoboken: John Wiley and Sons.

Lomas, T. (2017). Recontextualising mindfulness: Theravada Buddhist perspectives on the ethical and spiritual dimensions of awareness. Psychology of Religion and Spirituality, 9(2), 209-219. https://doi. org/10.1037/rel0000080.

Lomas, T., Cartwright, T., Edginton, T., \& Ridge, D. (2013). 'I was so done in that I just recognized it very plainly, "you need to do something"': men's narratives of struggle, distress and turning to meditation. Health, 17(2), 191-208. https://doi.org/10.1177/ 1363459312451178.

Lomas, T., Cartwright, T., Edginton, T., \& Ridge, D. (2014a). A religion of wellbeing?: the appeal of Buddhism to men in London, UK. Psychology of Religion and Spirituality, 6(3), 198-207. https://doi. org/10.1037/a0036420.

Lomas, T., Cartwright, T., Edginton, T., \& Ridge, D. (2014b). Engagement with meditation as a positive health trajectory: divergent narratives of progress in male meditators. Psychology and Health, 29(2), 218-236. https://doi.org/10. 1080/08870446.2013.843684.

Lomas, T., Cartwright, T., Edginton, T., \& Ridge, D. (2015a). A qualitative analysis of experiential challenges associated with meditation practice. Mindfulness, 6(4), 848-860. https://doi.org/10.1007/ s12671-014-0329-8.

Lomas, T., Cartwright, T., Edginton, T., \& Ridge, D. (2016). New ways of being a man: 'positive' hegemonic masculinity in meditation-based communities of practice. Men and Masculinities, 19(3), 289-310. https://doi.org/10.1177/1097184X15578531.

Lomas, T., Hefferon, K., \& Ivtzan, I. (2015b). The LIFE model: a metatheoretical conceptual map for applied positive psychology. Journal of Happiness Studies, 16(5), 1347-1364. https://doi.org/10.1007/ s10902-014-9563-y.

Lomas, T., Ivtzan, I., \& Fu, C. (2015c). A systematic review of the neurophysiology of mindfulness on EEG oscillations. Neuroscience \& Biobehavioral Reviews, 57, 401-410. https://doi.org/10.1016/j. neubiorev.2015.09.018.

Lomas, T., Medina, J. C., Ivtzan, I., Rupprecht, S., \& Eiroa-Orosa, F. J. (2017a). The impact of mindfulness on the wellbeing and performance of educators: a systematic review of the empirical literature. Teaching and Teacher Education, 61, 132-141. https://doi.org/10. 1016/j.tate.2016.10.008.

Lomas, T., Medina, J. C., Ivtzan, I., Rupprecht, S., \& Eiroa-Orosa, F. J. (2018). A systematic review of the impact of mindfulness on the wellbeing of healthcare professionals. Journal of Clinical Psychology, 7(3), 319-355. https://doi.org/10.1002/jclp.22515.

Lomas, T., Medina, J. C., Ivtzan, I., Rupprecht, S., Hart, R., \& EiroaOrosa, F. J. (2017b). The impact of mindfulness on wellbeing and performance in the workplace: an inclusive systematic review of the empirical literature. European Journal of Work and Organizational Psychology, 26(4), 492-513. https://doi.org/10.1080/1359432X. 2017.1308924

Luken, M., \& Sammons, A. (2016). Systematic review of mindfulness practice for reducing job burnout. American Journal of Occupational Therapy, 70(2). https://doi.org/10.5014/ajot. 2016.016956.

*Mackenzie, C. S., Poulin, P. A., \& Seidman-Carlson, R. (2006). A brief mindfulness-based stress reduction intervention for nurses and nurse aides. Applied Nursing Research, 19(2), 105-109. https://doi.org/ 10.1016/j.apnr.2005.08.002.

Mannion, R. (2014). Enabling compassionate healthcare: perils, prospects and perspectives. International Journal of Health Policy and Management, 2(3), 115-117. https://doi.org/10. 15171/ijhpm.2014.34.

*Manotas, M., Segura, C., Eraso, M., Oggins, J., \& McGovern, K. (2014). Association of brief mindfulness training with reductions in perceived stress and distress in Colombian health care professionals. International Journal of Stress Management, 21(2), 207225. https://doi.org/10.1037/a0035150.

*Martín-Asuero, A., \& García-Banda, G. (2010). The mindfulness-based stress reduction program (MBSR) reduces stress-related psychological distress in healthcare professionals. Spanish Journal of Psychology, 13(2), 897-905.

*Martín-Asuero, A., Queraltó, J. M., Pujol-Ribera, E., Berenguera, A., Rodriguez-Blanco, T., \& Epstein, R. M. (2014). Effectiveness of a mindfulness education program in primary health care professionals: a pragmatic controlled trial. Journal of Continuing Education in the Health Professions, 34(1), 4-12. https://doi.org/ 10.1002/chp.21211.

*McConachie, D. A. J., McKenzie, K., Morris, P. G., \& Walley, R. M. (2014). Acceptance and mindfulness-based stress management for support staff caring for individuals with intellectual disabilities. Research in Developmental Disabilities, 35(6), 1216-1227. https:// doi.org/10.1016/j.ridd.2014.03.005. 
McConville, J., McAleer, R., \& Hahne, A. (2017). Mindfulness training for health profession students - the effect of mindfulness training on psychological well-being, learning and clinical performance of health professional students: a systematic review of randomized and non-randomized controlled trials. EXPLORE: The Journal of Science and Healing, 13(1), 26-45. https://doi.org/10.1016/j. explore.2016.10.002.

Moher, D., Liberati, A., Tetzlaff, J., \& Altman, D. G. (2009). Preferred Reporting Items for Systematic Reviews and Meta-Analyses: the PRISMA statement. PLoS Medicine, 6(7), e1000097. https://doi. org/10.1371/journal.pmed.1000097.

National Collaborating Centre for Methods and Tools. (2008). Quality Assessment Tool For Quantiative Studies (QATQS). Hamilton: McMaster University.

*Newsome, S., Waldo, M., \& Gruszka, C. (2012). Mindfulness group work: preventing stress and increasing self-compassion among helping professionals in training. Journal for Specialists in Group Work, 37(4), 297-311. https://doi.org/10.1080/ 01933922.2012.690832.

Noetel, M., Ciarrochi, J., Van Zanden, B., \& Lonsdale, C. (2017). Mindfulness and acceptance approaches to sporting performance enhancement: a systematic review. International Review of Sport and Exercise Psychology, 1-37.

*Noone, S. J., \& Hastings, R. P. (2010). Using acceptance and mindfulness-based workshops with support staff caring for adults with intellectual disabilities. Mindfulness, 1(2), 67-73. https://doi. org/10.1007/s12671-010-0007-4.

*Pflugeisen, B. M., Drummond, D., Ebersole, D., Mundell, K., \& Chen, D. (2016). Brief video-module administered mindfulness program for physicians: a plot study. EXPLORE: The Journal of Science and Healing, 12(1), 50-54. https://doi.org/10.1016/j.explore.2015.10.005.

*Pipe, T. B., Bortz, J. J., Dueck, A., Pendergast, D., Buchda, V., \& Summers, J. (2009). Nurse leader mindfulness meditation program for stress management: a randomized controlled trial. Journal of Nursing Administration, 39(3), 130-137.

Pollard, E. L., \& Davidson, L. (2001). Foundations of child wellbeing. Paris: UNESCO.

*Poulin, P. A., Mackenzie, C. S., Soloway, G., \& Karayolas, E. (2008). Mindfulness training as an evidenced-based approach to reducing stress and promoting well-being among human services professionals. International Journal of Health Promotion and Education, 46(2), 72-80. https://doi.org/10.1080/14635240.2008.10708132.

*Raab, K., Sogge, K., Parker, N., \& Flament, M. F. (2015). Mindfulness-based stress reduction and self-compassion among mental healthcare professionals: a pilot study. Mental Health, Religion and Culture, 18(6), 503-512. https://doi.org/10.1080/ 13674676.2015.1081588.

R Core Team (2017). R: a language and environment for statistical computing. Vienna, Austria: R Foundation for Statistical Computing. Retrieved from https://www.R-project.org/.

Riley, R. D., Higgins, J. P. T., \& Deeks, J. J. (2011). Interpretation of random effects meta-analyses. BMJ, 342 (feb10 2), d549-d549. https://doi.org/10.1136/bmj.d549.

*Rimes, K. A., \& Wingrove, J. (2011). Pilot study of mindfulness-based cognitive therapy for trainee clinical psychologists. Behavioural and Cognitive Psychotherapy, 39(2), 235-241. https://doi.org/10.1017/ S1352465810000731.

Rudaz, M., Twohig, M. P., Ong, C. W., \& Levin, M. E. (2017). Mindfulness and acceptance-based trainings for fostering self-care and reducing stress in mental health professionals: a systematic review. Journal of Contextual Behavioral Science, 6(4), 380-390. https://doi.org/10.1016/j.jcbs.2017.10.001.

Salovey, P., \& Mayer, J. D. (1990). Emotional intelligence. Imagination, Cognition, and Personality, 9(3), 185-211.

Schwarzer, G. (2007). Meta: an R package for meta-analysis. $R$ News, $7(3), 40-45$.
Sedgwick, P., \& Marston, L. (2013). Meta-analyses: standardised mean differences. BMJ, 347(dec06 1), f7257-f7257. https://doi.org/10. 1136/bmj.f7257.

Segal, Z. V., Williams, J. M. G., \& Teasdale, J. D. (2002). Mindfulnessbased cognitive therapy for depression: a new approach to preventing relapse. New York: Guilford Press.

Seligman, M. E. P., \& Csikszentmihalyi, M. (2000). Positive psychology: an introduction. American Psychologist, 55, 5-14.

*Shapiro, S. L., Astin, J. A., Bishop, S. R., \& Cordova, M. (2005). Mindfulness-based stress reduction for health care professionals: results from a randomized trial. International Journal of Stress Management, 12(2), 164-176. https://doi.org/10.1037/1072-5245.12.2.164.

*Shapiro, S. L., Brown, K. W., \& Biegel, G. M. (2007). Teaching selfcare to caregivers: effects of mindfulness-based stress reduction on the mental health of therapists in training. Training and Education in Professional Psychology, 1(2), 105-115. https://doi.org/10.1037/ 1931-3918.1.2.105.

Shapiro, S. L., Carlson, L. E., Astin, J. A., \& Freedman, B. (2006). Mechanisms of mindfulness. Journal of Clinical Psychology, 62(3), 373-386. https://doi.org/10.1002/jclp.20237.

*Shapiro, S. L., Schwartz, G. E., \& Bonner, G. (1998). Effects of mindfulness-based stress reduction on medical and premedical students. Journal of Behavioral Medicine, 21(6), 581-599. https://doi. org/10.1023/A:1018700829825.

*Singh, N. N., Lancioni, G. E., Karazsia, B. T., Myers, R. E., Winton, A. S. W., Latham, L. L., \& Nugent, K. (2015). Effects of training staff in MBPBS on the use of physical restraints, staff stress and turnover, staff and peer injuries, and cost effectiveness in developmental disabilities. Mindfulness, 6(4), 926-937. https://doi.org/10.1007/ s12671-014-0369-0.

*Singh, N. N., Singh, S. D., Sabaawi, M., Myers, R. E., \& Wahler, R. G. (2006). Enhancing treatment team process through mindfulnessbased mentoring in an inpatient psychiatric hospital. Behavior Modification, 30(4), 423-441. https://doi.org/10.1177/ 0145445504272971.

Smith, M. L. (1980). Publication bias and meta-analysis. Evaluation in Education, 4(Supplement C), 22-24. https://doi.org/10.1016/0191765X(80)90004-X.

Sochos, A., Bowers, A., \& Kinman, G. (2012). Work stressors, social support, and burnout in junior doctors: exploring direct and indirect pathways. Journal of Employment Counseling, 49(2), 62-73. https://doi.org/10.1002/j.2161-1920.2012.00007.x.

*Song, Y., \& Lindquist, R. (2015). Effects of mindfulness-based stress reduction on depression, anxiety, stress and mindfulness in Korean nursing students. Nurse Education Today, 35(1), 86-90. https://doi. org/10.1016/j.nedt.2014.06.010.

Spielmans, G. I., \& Flückiger, C. (2018). Moderators in psychotherapy meta-analysis. Psychotherapy Research, 28(3), 333-346. https:// doi.org/10.1080/10503307.2017.1422214.

Tang, Y.-Y., Ma, Y., Wang, J., Fan, Y., Feng, S., Lu, Q., ... Posner, M. I. (2007). Short-term meditation training improves attention and selfregulation. Proceedings of the National Academy of Sciences, 104(43), 17152-17156. https://doi.org/10.1073/pnas.0707678104.

The Health \& Social Care Information Centre. (2009). Adult psychiatric morbidity in England: results of a household survey. London: The Health \& Social Care Information Centre.

Toppinen-Tanner, S., Ojajärvi, A., Väänaänen, A., Kalimo, R., \& Jäppinen, P. (2005). Burnout as a predictor of medically certified sick-leave absences and their diagnosed causes. Behavioral Medicine, 31(1), 18-32. https://doi.org/10.3200/BMED.31.1.18-32.

Trowbridge, K., \& Mische Lawson, L. (2016). Mindfulness-based interventions with social workers and the potential for enhanced patient-centered care: a systematic review of the literature. Social Work in Health Care, 55(2), 101-124. https://doi.org/10.1080/00981389.2015.1094165.

Van Gordon, W., Shonin, E., Lomas, T., \& Griffiths, M. D. (2016). Corporate use of mindfulness and authentic spiritual transmission: 
competing or compatible ideals? Mindfulness and Compassion, 1(2), 75-83. https://doi.org/10.1016/j.mincom.2016.10.005.

Vettese, L. C., Toneatto, T., Stea, J. N., Nguyen, L., \& Wang, J. J. (2009). Do mindfulness meditation participants do their homework? And does it make a difference? A review of the empirical evidence. Journal of Cognitive Psychotherapy, 23(3), 198-225. https://doi. org/10.1891/0889-8391.23.3.198.

Virgili, M. (2015). Mindfulness-based interventions reduce psychological distress in working adults: a meta-analysis of intervention studies. Mindfulness, 6(2), 326-337. https://doi.org/10.1007/s12671-013-0264-0.

Vogus, T. J., Cooil, B., Sitterding, M., \& Everett, L. Q. (2014). Safety organizing, emotional exhaustion, and turnover in hospital nursing units. Medical Care Research and Review, 52(10), 870-876.

Walsh, R., \& Shapiro, S. L. (2006). The meeting of meditative disciplines and western psychology: a mutually enriching dialogue. American Psychologist, 61(3), 227-239. https://doi.org/ 10.1037/0003-066X.61.3.227.
Weng, H.-C., Hung, C.-M., Liu, Y.-T., Cheng, Y.-J., Yen, C.-Y., Chang, C.-C., \& Huang, C.-K. (2011). Associations between emotional intelligence and doctor burnout, job satisfaction and patient satisfaction. Medical Education, 45(8), 835-842. https://doi.org/10.1111/j. 1365-2923.2011.03985.x.

*West, C. P., Dyrbye, L. N., Rabatin, J. T., Call, T. G., Davidson, J. H., Multari, A., ... Shanafelt, T. D. (2014). Intervention to promote physician well-being, job satisfaction, and professionalism a randomized clinical trial. JAMA Internal Medicine, 174(4), 527-533. https://doi.org/10.1001/jamainternmed.2013.14387.

Wolever, R. Q., Bobinet, K. J., McCabe, K., Mackenzie, E. R., Fekete, E., Kusnick, C. A., \& Baime, M. (2012). Effective and viable mindbody stress reduction in the workplace: a randomized controlled trial. Journal of Occupational Health Psychology, 17(2), 246-258. https://doi.org/10.1037/a0027278. 